\title{
Chiarimenti pontifici sul "processus brevior". Riflessioni alla luce del Discorso del 25 novembre 2017
}

\section{Pontifical Clarifications Regarding the "processus brevior". Reflections in Light of the Address on November 25, 2017}

\author{
Massimo DEL Pozzo \\ Profesor Extraordinario de Derecho Constitucional Canónico \\ Pontificia Università della Santa Croce. Facoltà di Diritto Canonico. Roma \\ orcid 0000-0003-4816-1965 \\ delpozzo@pusc.it
}

Resumen: La presente contribución, partiendo del contexto y de la articulación global del Discurso del Papa del 25 de noviembre de 2017, analiza principalmente las aclaraciones pontificias relativas al processus brevior. La llamada al ejercicio personal y exclusivo de la potestad judiciaria del Obispo en el processus brevior pretende estimular la apropiada puesta en práctica de un principio cardinal de la reforma (la efectiva centralidad del Obispo en la administración de la justicia matrimonial). Quedando intacto el valor de una suerte de interpretación auténtica del texto hecha "a viva voz", las indicaciones pontificias (expresadas en 9 puntos) deben ser tenidas en consideración según su tenor y contenido. La intervención pontificia constituye una nueva contribución en el camino de la reforma que no altera ni trastorna el texto legal ni los principios del sistema. La respuesta a las puntualizaciones interpretativas papales dependerá del efectivo concurso del cuerpo episcopal, de la profundización científica y de la coordinación institucional.

Palabras clave: Motu Proprio Mitis iudex Dominus lesus, processus brevior coram Episcopo, Obispo diocesano juez, Aclaraciones pontificias (Discurso del 25 de noviembre de 2017).
Abstract: Starting from an overview of the context and overall message of the Papal Address on November 25, 2017, this article analyzes the Pope's clarifications of the processus brevior in particular. The reference made to the personal and exclusive exercise of the judicial power of the Bishop in the briefer process requires the implementation of one of the reform's key principles (the central role of the Bishop in the administration of matrimonial justice). Without prejudice to the value of an authentic "viva voce" interpretation of the text, given their content and import the Pope's clarifications (expressed in nine points) ought to be taken into account. The pontifical address represents a further contribution to the path of the reform that neither alters nor overturns the legal text itself nor the principles of the system. Ultimately, feedback regarding these interpretive papal clarifications depends on effective collaboration from the episcopacy, ongoing academic study, and coordination at an institutional level.

Keywords: Motu Proprio Mitis iudex Dominus lesus, processus brevior coram Episcopo, Diocesan Bishop as Judge, Pontifical Clarifications (Address on November 25, 2017). 


\section{Il valore e l'intento dell'intervento del PAPA E LO SPIRITO DEL PRESENTE CONTRIBUTO}

$\mathrm{n}$ occasione dell'udienza conclusiva del Corso sul nuovo processo matrimoniale e la procedura super rato promosso dal Tribunale della Rota Romana il Santo Padre ha voluto proporre delle considerazioni sullo spirito della riforma e fornire delle puntuali prescrizioni interpretative sul processus matrimonialis brevior coram Episcopo ${ }^{1}$. Il Discorso pertanto non ha il semplice carattere di delucidazione concettuale e puntualizzazione direttiva circa l'oggetto dell'incontro (come sono soliti essere gli interventi pontifici in simili occasioni) ma ha un contenuto prescrittivo e, in un certo senso, normativo in merito all'impianto dei Motu proprio e al processus brevior in specie. La riunione ha fornito dunque lo spunto per cercare di superare dubbi o impasse nell'attuazione del disposto legislativo. L'intervento merita pertanto adeguata attenzione e matura riflessione per dare concreto riscontro alle indicazioni pontificie.

Il contesto e la forma adottati (discorso o allocuzione anziché un provvedimento formale) nulla tolgono ovviamente al significato e al valore del testo pronunciato. La modalità prescelta può comportare evidentemente dei limiti per la conoscibilità e diffusione nonché per la redazione tecnica della prolusione ma non altera o stravolge i principi del sistema ${ }^{2}$. È nella logica dell'autorità suprema scegliere discrezionalmente (pro bono Ecclesiae) la tipologia della risoluzione. In assenza di ogni autolimitazione formale, la comunicazione espressa e diretta della determinazione esplicativa del Papa ha evidentemente l'autorevolezza e rilevanza di un atto del Supremo Legislatore. La scelta operata elimina così ogni riserva o perplessità circa la valenza dell'eventuale attività interpretativa o istruttiva di altri organi ${ }^{3}$. Anche nell'attuazione della riforma non sono

${ }^{1}$ Francesco, Discorso ai partecipanti al corso promosso dal Tribunale della Rota Romana, 25-XI-2017 [= Discorso]. Le citazioni riportate nel testo («...»), senza altra indicazione, si riferiscono a tale Discorso, per l'articolato si indicano i numeri (n.) e per la parte introduttiva i capoversi (cpv.). Non si riporta la localizzazione dei documenti agevolmente reperibili nel sito www.vatican.va.

2 I problemi accennati si riferiscono alla promulgazione dell'atto e alla problematica conciliazione del tono caldo e paterno di un'udienza pontificia con il rigore e la nettezza di una precisazione ermeneutica in un ambito tecnicamente assai complesso. Sul principio di legalità circa le modalità di produzione e promulgazione normativa, cfr. J. LlObeLL, Processi e procedure penali: sviluppi recenti, in H. FRANCESCHI - M. Á. ORTIZ (eds.), Ius et matrimonium. Temi di diritto matrimoniale e processuale canonico, EDUSC, Roma 2015, 79-82.

3 Com'è noto, l'istr. Dignitas connubii ha suscitato inizialmente qualche dubbio o difficoltà circa il suo valore normativo, cfr. ad es. E. BAURA, Il valore normativo dell'Istruzione "Dignitas connubii", in 
mancate peraltro incertezze e taluni malumori circa la natura e l'opportunità di provvedimenti atipici o poco lineari ${ }^{4}$. Il Santo Padre ha ritenuto pertanto di evitare ambiguità o resistenze attraverso una disposizione "viva voce" della sua determinazione operativa. Ł̀ stato già chiarito che il magistero del Pontefice ha un immediato rilievo giuridico e normativo nella misura in cui interessa la giustizia delle relazioni ecclesiali senza necessità di alcun atto di positivizzazione o formalizzazione ${ }^{5}$. Un discorso diverso (inerente la prudenza e il discernimento del Legislatore) concerne l'ordine e congruenza delle fonti legislative $^{6}$. In questo caso si tratta quindi di una serie di interpretazioni e di raccomandazioni "autentiche" del Papa (in seguito esploreremo meglio la portata disciplinare complessiva del Discorso).

Il fine dell'intervento del Santo Padre sembra quello di sollecitare la retta applicazione della riforma. Il numero e l'ampiezza delle questioni affrontate rende tuttavia più difficile e ostico il lavoro dell'interprete ${ }^{7}$. Alla determinazione delle coordinate del disegno legislativo (spirito sinodale e consolazione pastorale), si associa una specifica puntualizzazione risolutoria circa il processo breviore. Il cuore o nucleo del Discorso, soprattutto da un punto di vista dispositivo, sta nel richiamo all'obbligatorietà e al senso del processus brevior. Un serio problema nell'attuazione della riforma sembra legato infatti alla disapplicazione e renitenza oppure alla indebita delega o sbrigatività nell'esercizio della funzione giudiziaria da parte di alcuni Vescovi in riferimento a questa figura procedimentale $^{8}$. Al Successore di Pietro non interessa tanto il concreto in-

Il giudizio di nullità matrimoniale dopo l'istruzione «Dignitas connubii». Parte Prima: $i$ principi, P. A. BONNET - C. Gullo (eds.), LEV, Città del Vaticano 2007, 185-211; J. Llobell, La natura giuridica e la recezione ecclesiale dell'istr. «Dignitas connubii», Ius Ecclesiae 18 (2006) 344-354.

${ }^{4}$ Si pensi ad es., per quanto abbia un valore didascalico e formativo, alle affermazioni contenute in Tribunale Apostolico della Rota Romana, Sussidio applicativo del Motu pr. Mitis Iudex Dominus Iesus, Città del Vaticano, gennaio 2016 [= Sussidio applicativo MIDI] o all'estensione dei rilievi contenuti in Conferenza Episcopale Italiana, Testo del tavolo di lavoro, 20-VII-2016, [= Tavolo di lavoro CEI].

5 Cfr. J. Llobell, Sulla valenza giuridica dei discorsi del Romano Pontefice al Tribunale Apostolico della Rota Romana, L'Osservatore Romano 6-XI-2005, 7-8.

${ }^{6}$ Le possibili disquisizioni sulla rispondenza o avvedutezza della pratica non inficiano certo la validità e vigenza del disposto incontrovertibilmente ex audientia Sanctissimi.

${ }^{7}$ In seguito ci soffermeremo anche sulla disparità e disomogeneità del tenore dei richiami (infra $\$ \$ 3-6)$.

8 E indicativo il riscontro contenuto in L. Musso - C. FusCo (eds.), La riforma del processo matrimoniale ad un anno dal Motu Proprio Mitis Iudex Dominus Iesus, LEV, Città del Vaticano 2017, 173-193, ove si prospettano indicazioni sulle realtà statunitense e tedesca, rileva ad es. E. Glaubitz, giudice del Tribunale di Colonia: «La maggior parte dei vescovi tedeschi non ha intenzione di attuare questa proposta [...] Non mi consta che in questo primo anno di attuazione del Motu 
centivo di questa forma processuale (i presupposti sono presentati anzi in maniera ancor più rigorosa ${ }^{9}$ ) quanto la effettiva sensibilità e la disponibilità dei Pastori per l'amministrazione di una giustizia matrimoniale sollecita e vicina ai propri fedeli. Non pretendiamo ovviamente di ricostruire la motivazione dell'intervento pontificio, peraltro abbastanza esplicita e chiara, cerchiamo solo di inquadrarlo e contestualizzarlo. Il Papa ha voluto dunque offrire riferimenti e orientamenti a tutti gli operatori, correggere o prevenire fraintendimenti ed equivoci applicativi e dare istruzioni e indicazioni ai fratelli nell'Episcopato. Lo spirito dell'iniziativa si ripercuote d'altronde sulla percezione del significato delle affermazioni. Interessa sottolineare che il Discorso anche quoad processu breviore non può essere avulso o isolato dalle prescrizioni codiciali ${ }^{10}$. Il Santo Padre non ci pare abbia inteso innovare o modificare il dettato del Mitis iudex, ha voluto precisarlo e puntualizzarlo. Il testo va pertanto letto e interpretato sempre nel contesto della riforma.

Il presente contributo non rappresenta pertanto una sorta di glossa o notazione a margine del Discorso, ma un tentativo di esposizione o, piuttosto, di ricostruzione delle indicazioni e prescrizioni ivi contenute per coglierne e valorizzarne l'apporto direttivo o normativo. All'opera di enucleazione e concettualizzazione delle possibili istruzioni, si associa soprattutto quella di analisi ed esegesi del disposto. Precisiamo subito che la spiegazione non è sempre immediata e semplice ${ }^{11}$. Il nostro impegno ermeneutico cerca in genere di inserire e coniugare il Discorso nell'impianto codiciale, già altrove tratteggiato ${ }^{12}$, ci auguriamo, senza mai stravolgere o alterare la sostanza delle affermazioni pontificie. Le precisazioni del Papa obbligano comunque ad alcuni doverosi ripensamenti e ritrattazioni ${ }^{13}$. Come ribadiremo meglio conclusivamente (infra $\$ 7$ ), si tratta di una riflessione aperta e necessariamente provvisoria.

Proprio nessun vescovo tedesco sia stato giudice di un processo più breve e neanche che sia stato mai richiesto tale processo» (189), talune riserve, con una maggior propensione sono state formulate anche per gli Stati Uniti (179-180, 182).

$\left.9 \begin{array}{ll} & \ll\end{array} . ..\right]$ richiedendosi come condizione imprescindibile [del processo breviore] l'assoluta evidenza dei fatti comprovanti la presunta nullità del coniugio, oltre al consenso dei due sposi». L'evidenza viene qui definita "assoluta" in merito, infra $\$ 7$.

${ }^{10}$ Cfr. anche can. $1691 \$ 3$.

${ }^{11}$ Un passo ad es. che ci risulta piuttosto ostico è quello che precede la benedizione finale: «In conclusione, vorrei ribadire con chiarezza che ciò avviene senza chiedere il permesso o l'autorizzazione ad altra Istituzione oppure alla Segnatura Apostolica».

12 Cfr. M. DEL Pozzo, Il processo matrimoniale più breve davanti al Vescovo, EDUSC, Roma 2016.

${ }^{13}$ Spicca subito la necessità del requisito della consacrazione episcopale per l'individuazione del giudice, cfr. M. DEL POZZO, Il processo matrimoniale più breve..., cit., 59-64. 


\section{Lo SVILUPPo E L'ARTICOLAZIONE DEL DisCORSO PAPALE}

Il Discorso papale si può dividere idealmente in due parti: 1) l'inquadramento e motivazione della riforma; 2) la precisazione autoritativa di alcuni aspetti del processo breviore. Lo scopo precettivo dei chiarimenti e, in un certo senso, lo stacco rispetto alla sezione più discorsiva e illustrativa è dimostrato, oltre che dalla solennità dell'introduzione («ho deciso, in ragione dell'ufficio di Vescovo di Roma e Successore di Pietro, di precisare definitivamente alcuni aspetti fondamentali dei due Motu proprio»), anche dalla formulazione (stringata e tecnica) e dall'articolazione (per punti) delle delucidazioni. Benché l'interesse principale di questo contributo è riservato agli espliciti chiarimenti sul processus brevior, riteniamo imprescindibile un riferimento ai contenuti di fondo o più generali dell'insegnamento del Pontefice. Il Santo Padre ha auspicato infatti che lo spirito sinodale e la consolazione pastorale conformino l'attività degli operatori, in particolare nella «ricerca della verità sullo stato coniugale dei coniugi». A questi due elementi riteniamo utile aggiungere anche il richiamo pregiudiziale al ruolo del Vescovo che è alla base dell'attuazione della forma breviore.

Il Papa ha sottolineato con forza e determinazione la natura sinodale dei Motu Proprio adottati ${ }^{14}$ : «Questi due provvedimenti sono scaturiti da un contesto sinodale, sono espressione di un metodo sinodale, sono l'approdo di un serio cammino sinodale». La sinodalità è quindi colta a livello genetico, metodologico e teleologico. Lo spirito sinodale viene anzi indicato come prassi o stile (dialogico e complessivo) che dovrebbe connotare sempre di più l'azione della Chiesa ${ }^{15}$. La tematica ha stimolato già un certo dibattito che, al di là degli stimoli e delle suggestioni intellettuali e operative, riguarda soprattutto le scelte e i contenuti del governo ecclesiastico ${ }^{16}$. A fronte della novità e incisività dell'intervento legislativo, non sono mancate peraltro riserve o polemiche

${ }^{14}$ Cfr. Francesco, litterae ap. motu proprio datae Mitis iudex Dominus Iesus, 15 agosto 2015 [= MIDI]; IDEM, m. p. Mitis et Misericors Iesus, 15 agosto 2015.

15 Cfr. ad es. Francesco, es. ap. Evangeli gaudium, 23 novembre 2013, n. 31; Discorso per la commemorazione del $50^{\circ}$ anniversario dell'istituzione del Sinodo dei Vescovi, 17 ottobre 2015; Lettera al Card. Marc Ouellet, Presidente della Pontificia Commissione per l'America latina, 19 marzo 2016.

${ }^{16}$ Cfr. L. BAdisseri (ed.), A cinquant'anni dall'Apostolica Sollicitudo. Il Sinodo dei Vescovi al servizio di una Chiesa sinodale. Atti del Seminario di studio organizzato dalla Segreteria generale del Sinodo dei Vescovi (Città del Vaticano, 6-9 febbraio 2016), LEV, Città del Vaticano 2016. Interessanti sono pure le considerazioni di A. VIANA, Sinodalidad y derecho canónico. Un seminario organizado por la Secretaría general del Sínodo de los Obispos, Estudios eclesiásticos 92 (2017) 683-701. 
circa l'istanza e l'effettivo coinvolgimento episcopale ${ }^{17}$. Il Santo Padre ha pertanto voluto ribadire con chiarezza e fermezza l'origine e il criterio ermeneutico che anima la normativa: soddisfare le aspettative di giustizia del popolo cristiano circa l'accertamento della nullità matrimoniale ${ }^{18}$. La consultazione $\mathrm{e}$ la riflessione dell'assise sinodale ha fornito la base d'ispirazione della riforma codiciale. La matrice sinodale ovviamente non risiede tanto nella redazione o conformazione del testo legislativo (demandata, com'è noto, alla Commissione istituita e agli esperti consultati ${ }^{19}$ ), quanto nei motivi e nelle richieste palesate. Parecchi autori hanno già insistito sull'esigenza di inserire il testo nel contesto di gestazione e remota provenienza e, soprattutto, di integrare la riforma nella spinta pastorale familiare impressa dai Sinodi $i^{20}$. Questo indirizzo ermeneutico riceve insomma un ulteriore autorevole avallo. L'obiettivo del Papa non pare comunque teoretico, ma pratico: favorire l'applicazione fedele delle nuove procedure, evitando critiche o contestazioni pretestuose.

L'altro principio direttivo espressamente additato dal Pontefice è la consolazione pastorale. La comprensione e vicinanza ecclesiale e la preoccupazione per la salvezza e pace delle coscienze rappresenta espressamente la finalità della nuova normativa processuale matrimoniale ${ }^{21}$. Nelle parole del Papa la sofferenza, la solitudine, il tormento dei coniugi che hanno sperimentato un fallimento matrimoniale costituiscono motivo di speciale attenzione e sollecitudine per gli operatori dei tribunali ecclesiastici. Il profilo pastorale definis-

17 Cfr. G. BoNI, La recente riforma del processo di nullità matrimoniale. Problemi, criticità, dubbi (parte prima), Stato, Chiese e pluralismo confessionale. Rivista telematica (www.statoechiese.it) n. 9 (2016) 57-71; G. RabiNo, Riflessioni sull'origine sinodale del processus brevior coram Episcopo, Stato, Chiese e pluralismo confessionale. Rivista telematica (www.statoechiese.it) n. 33 (2017) 1-24.

18 Cfr. anche FranCESCO, Discorso ai partecipanti al corso promosso dal Tribunale della Rota Romana, 12 marzo 2016, Quaderni dello Studio rotale 9 (2016) 49-52 (nella pubblicazione si inserisce la parte a braccio non riportata nel sito); IDEM, Conferenza stampa durante il volo di ritorno dagli Stati Uniti d'America, 27 settembre 2015.

19 Per la nomina e il mandato alla Commissione pontificia incaricata cfr. Nota 27 agosto 2014 in Comunicato della Sala Stampa della Santa Sede, 20 settembre 2014. La ricostruzione dei diversi passaggi della riforma è proposta anche da A. W. Bunge, Presentación del nuevo proceso matrimonial, Anuario Argentino de Derecho Canónico 21 (2015) 73-75.

${ }^{20}$ Cfr. ad es. M. J. ARroba CONDE - C. IzZI, Pastorale giudiziaria e prassi processuale nelle cause di nullità del matrimonio. Dopo la riforma operata con il motu proprio Mitis Iudex Dominus Iesus, Edizioni San Paolo, Cinisello Balsamo 2017, 47-62; G. BELFIORE, I processi di nullità matrimoniale nella riforma di papa Francesco, Edizioni Grafiser-Troina, Catania 2017, 7-48; M. DEL PozZO, Il processo matrimoniale più breve..., cit., 19-25.

21 Cfr. anche Francesco, Discorso in occasione dell'inaugurazione dell'anno giudiziario del Tribunale della Rota Romana, 29-I-2018, con commento M. DEL POZZO, La centralità della coscienza nella verità del matrimonio, Ius Ecclesiae 30 (2018) 367-380. 
ce il «giusto approccio alla questione», il valore aggiunto di umanità e comprensione, che connota il sistema ecclesiale. Il processo e in generale il servizio giudiziario si possono trasformare così in occasione di incontro con la Grazia e di riscoperta dell'Amore divino. La consolazione pastorale non è però un surrogato o un palliativo della verità e della giustizia per rimediare in maniera lassista e arrendevole agli errori e alle delusioni della vita coniugale. Alla sensibilità caritatevole degli agenti si associa appunto lo studio serio e profondo dei casi e della legislazione. Secondo Francesco la sfida ecclesiale consiste nel coniugare l'accoglienza e la cura delle persone ferite con «la difesa della sacralità del vincolo matrimoniale» ${ }^{22}$. Non bisogna pertanto confondere l'incremento nello stile e nel metodo degli operatori con cedimenti e rilassamenti nella scienza e nella coscienza dei giudici e dei coniugi.

Un terzo spunto che ci sembra utile evidenziare è l'enfasi sulla centralità della funzione episcopale. Il Papa non indica il ruolo del Vescovo, come per i precedenti due concetti, quale orizzonte assiologico della novella codiciale (origine e fine ${ }^{23}$ ), lo individua tuttavia come un cardine attuativo della riforma. L'esemplarità e il riferimento capitale sono d'altronde l'indicazione più chiara e convincente del desiderio di conformazione e riforma personale ${ }^{24}$. L'invito alla collaborazione leale col proprio Vescovo aggiunge la notazione che ha un compito determinante «in quanto egli è il "giudice nato" della Chiesa particolare». Le precisazioni e le sollecitazioni operative vogliono recuperare dunque il senso e la portata dell'innovazione legislativa: la responsabilità diretta e personale del Vescovo nell'amministrazione della giustizia matrimoniale. L'impegno e la sensibilità complessiva probabilmente conta più della concreta implicazione nel giudizio breviore. Le questioni più specifiche e puntuali derivano allora da una preoccupazione di principio e d'indirizzo: evidenziare la natura intrinsecamente giudiziaria del munus episcopale. Le carenze o disfunzioni nel proces-

22 Ibíd.

23 «Ritornando nelle vostre comunità, sforzatevi di essere missionari e testimoni dello spirito sinodale che è all'origine di esse, come anche della consolazione pastorale che è il fine di questa nuova normativa matrimoniale, per corroborare la fede del popolo santo di Dio mediante la carità» $\left(4^{\circ}\right.$ cpv., Discorso).

${ }^{24}$ Lo stesso Papa Francesco non si è sottratto al compito giudicante quale Vescovo diocesano, sono state rese note almeno un paio di sentenze, cfr. ad es. P. V. PINTO, Matrimonio y familia en el camino sinodal del Papa Francisco, Arquidiócesis de Bogotá 2017, pro manuscripto, 278-283 (Processus brevior del Papa Francisco Obispo de Roma, 13-VII-2017); Romana coram Francisco P.P., sentenza, 16-V-2017, Prot. 18.067, in AA. Vv., Prassi e sfide dopo l'entrata in vigore del m.p. Mitis Iudex Dominus Iesus e del Rescriptum ex audientia del 7 dicembre 2015, LEV, Città del Vaticano 2017, 141-149. 
sus brevior minano o indeboliscono la credibilità della richiesta conversione istituzionale ${ }^{25}$. Più che rimediare alle mancanze o urgenze interessa in primis consolidare la "chiave di volta" dell'impianto giudiziario.

\section{LA FORMA E L'IMPOSTAZIONE DEI CHIARIMENTI PONTIFICI}

Precisato il fine e il contesto dell'intervento papale, conviene chiarire preliminarmente lo stile e il carattere dei chiarimenti pontifici circa il processus brevior, anche per cercare di far luce sull'apprezzamento delle diverse affermazioni. Non si può non notare una certa discrasia tra l'impostazione e la premessa autoritativa (decisionale e disciplinare più che dottrinale e magisteria$\mathrm{l}^{26}$ ) e l'atteggiamento e il contenuto di talune prescrizioni (sollecitatorie e orientative). Una nota che ci pare contraddistingua l'approccio del Papa, non soltanto nell'ambito della riforma processuale, è quello del pragmatismo operativo e della collaborazione istituzionale ${ }^{27}$. La delicatezza del rapporto con i Vescovi ha indotto il Pontefice, anche in linea con l'essenza interpretativa del Discorso, a preferire almeno nell'aspetto organizzativo la strada della direttiva e dell'orientamento a quella dell'ingiunzione e del comando.

Il tono dell'intervento, come anticipato, è particolarmente solenne e quasi risolutorio. $\mathrm{Al}$ di là della qualificazione primaziale dell'atto, nella formula già riportata, il Papa esplicita infatti la "definitività" e "fondamentalità" delle precisazioni fornite. Il chiarimento vorrebbe rendere insomma l'applicazione della nuova legge sul processo matrimoniale certa e sicura. Il tempo segnalato («a due anni dalla promulgazione» ${ }^{28}$ ) indica la maturazione intervenuta e la percezione dell'opportunità delle delucidazioni ${ }^{29}$. La manifestazione della voluntas, più che della semplice mens, del Legislatore intende in

$25 \ll$ Si auspica pertanto che nelle grandi come nelle piccole diocesi lo stesso Vescovo offra un segno della conversione delle strutture ecclesiastiche, e non lasci completamente delegata agli uffici della curia la funzione giudiziaria in materia matrimoniale» (III Criterio fondamentale, Proemio MIDI). La conversione richiesta ha sempre una dimensione personale, oltre che istituzionale.

${ }^{26}$ Cfr. G. GHIRLANDA, Il diritto nella Chiesa mistero di comunione. Compendio di diritto ecclesiale, Gregorian \& Biblical Press, Roma 2014, 650.

27 Il Tavolo di lavoro CEI può esserne una dimostrazione esemplare.

${ }^{28}$ Per una ricostruzione molto attenta ma abbastanza critica delle vicende che si sono succedute in questo periodo, cfr. G. BoNI, La riforma del processo canonico di nullità matrimoniale: il complicarsi progressivo del quadro delle fonti normative (parte prima, seconda e terza), Stato, Chiese e pluralismo confessionale. Rivista telematica (www.statoechiese.it) nn. 4-6 (2018) 1-78, 1-103, 1-29.

29 Quasi a voler indicare che, terminato il tempo del dibattito scientifico e del rodaggio operativo, è giunto il momento della corretta interpretazione e applicazione del disposto. 
pratica dissipare le confusioni, le titubanze e talune resistenze presenti nel corpo episcopale. Un'interpretazione autentica viva voce, oltretutto abbastanza dettagliata e articolata, d'altronde è un'operazione piuttosto insolita e singolare, non stupisce pertanto che anche l'approccio appaia risoluto e determinato ${ }^{30}$.

Fermo restando l'indubbia autorevolezza e l'imperatività delle affermazioni, riteniamo che s'imponga un'interpretazione razionale e sistematica delle disposizioni. In questa linea ci pare di dover cogliere dalla stessa formulazione letterale o dall'impianto logico delle asserzioni la diversa natura delle precisazioni e la variabilità del contenuto precettivo. Senza nulla togliere all'incipit della parte prescrittiva, dal testo si evincono criteri univoci e perentori d'immediata applicazione, spinte e solleciti d'azione aperti all'integrazione e determinazione dei singoli Vescovi, principi direttivi e assiologici di riferimento. L'interpretazione primaziale è insomma differenziata e variegata ex natura textus ${ }^{31}$. Bisogna pertanto distinguere le prescrizioni ermeneutiche immediatamente operative, dalle indicazioni sollecitatorie e dai richiami di principio. Questa puntualizzazione pregiudiziale non vuole sminuire il senso e la portata del Discorso pontificio, aspira anzi a rispettarne l'autentica valenza e rispondenza direttiva. Il riconoscimento lampante e incontrovertibile della «figura del Vescovo diocesano come giudice personale e unico nel Processo breviore» (che è il dichiarato proposito dei chiarimenti) peraltro non solo deve essere individuato e assicurato, ma, in larga misura, aspetta ancora di essere promosso e valorizzato secondo le aspirazioni del cammino sinodale ${ }^{32}$.

\section{LE DIRETTE PRESCRIZIONI ERMENEUTICHE}

Il primo ambito di rilevanza dell'intervento papale concerne $\mathrm{i}$ chiarimenti suscettibili di diretta e immediata applicazione. Parliamo di "prescrizioni ermeneutiche" perché le spiegazioni fornite hanno il valore un'interpre-

${ }^{30}$ Per quanto riteniamo che le garanzie formali siano importanti, si può ricondurre alla sostanzialità e determinazione del ruolo primaziale. Un caso simile è rappresentato dalla diretta interpretazione del m.p. Magnum principium, cfr. FranCESCO, Lettera al Card. Sarah, 15 ottobre 2017, in bttps://it.zenit.org/articles/papa-scrive-lettera-al-card-sarab-riguardo-al-motu-proprio-magnumprincipium/cons. 26/02/2018.

31 Non si tratta insomma di una semplice interpretazione o correzione puntuale ma di una più generale aspirazione chiarificatrice di guida e d'indirizzo.

32 Come spiegheremo meglio in seguito, conta anzitutto la disponibilità del ruolo e della figura, l'azione e l'attività in concreto svolta può essere magari modesta. 
tazione autentica del testo legale ${ }^{33}$. In un paio di casi il Santo Padre offre in realtà una giustificazione o ratio della scelta operata ${ }^{34}$, la puntualizzazione tuttavia contribuisce a precisare il senso delle disposizioni. Il valore prevalentemente dichiarativo delle statuizioni e l'incontrovertibilità dell'interpretazione proposta non ammette troppi dubbi o incertezze. Eventuali equivoci o, soprattutto, contestazioni porterebbero quindi a stravolgere il contenuto dell'insegnamento pontificio ${ }^{35}$. Non bisogna chiaramente confondere il riconoscimento e l'accettazione della vincolatività di una prescrizione dalle possibili riserve o apprezzamenti critici nel merito del decisum e dalle proposte de iure condendo. Occorre sempre percepire lo stacco tra il fatto giuridico o la dichiarazione normativa e il giudizio o l'opinione.

\subsection{L'esercizio personale ed esclusivo della giurisdizione decisoria nel processo breviore}

Lidea principale soggiacente ai chiarimenti pontifici è che il processus brevior non solo è riservato esclusivamente al Vescovo diocesano e al soggetto ad esso equiparato (l'individuazione del Giudice-Vescovo verrà approfondita tra poco, infra \$4.2), ma manifesta la pienezza della potestà giudiziaria dell'ufficio capitale. La forma procedurale non rientra quindi nelle prerogative o facoltà del Pastore ma nelle responsabilità o funzioni demandate a tale Vescovo. Nella determinazione della specifica competenza giudiziaria episcopale interessa sottolineare anzitutto i caratteri della personalità ed esclusività dell'attribuzione che fungono da cardine o fulcro delle prescrizioni dell'allocuzione: $\ll[\ldots]$ stabilisco di seguito quanto ritengo determinante ed esclusivo nell'esercizio personale del Vescovo diocesano giudice ${ }^{36}$. Non è casuale quindi che tali note vengano ribadite ripetutamente nel contesto del Discorso ${ }^{37}$. Il binomio (per-

${ }^{33}$ Cfr. in generale M. GANARIN, Linterpretazione autentica delle leggi universali della Chiesa, Bononia University Press, Bologna 2016; IDEM, Il Pontificio Consiglio per i Testi Legislativi nell'assetto istituzionale della Curia Romana tra diritto vigente e prospettive di riforma, Stato, Chiese e pluralismo confessionale. Rivista telematica (www.statoechiese.it) n. 22 (2015) 1-76.

${ }^{34}$ Il Papa fornisce una spiegazione a proposito della riserva episcopale della procedura (n. 4, Discorso) e della potestà del Decano della Rota (n. 9).

35 Ciò non significa che la determinazione della portata di un'affermazione non possa poi dar luogo a perplessità o contrasti dottrinali.

${ }^{36} 6^{\circ}$ cpv., Discorso.

$37 \ll[. .$.$] la figura del Vescovo diocesano come giudice personale ed unico nel Processo breviore» \left(6^{\circ} \mathrm{cpv}\right.$., Discorso); «Il Vescovo diocesano in forza del suo ufficio pastorale è giudice personale ed unico nel processo breviore» (n. 1); «La competenza esclusiva e personale del Vescovo diocesano, [...]» (n. 4). 
sonalità e unicità) che ricorre in tutti i riferimenti sottende una duplicità di accezioni che vale la pena di esplicitare.

La personalità della competenza procedurale statuita dal Papa indica l'esercizio in prima persona del titolare proprio della potestà giudiziaria nel processus brevior. Nel Proemio del MIDI era già contenuto un forte richiamo al significato della personalità della iurisdictio ${ }^{38}$. Non riteniamo che dal disposto legale potessero sorgere troppi dubbi circa l'esercizio diretto e monocratico della potestà giudicante nel processo breviore ${ }^{39}$. Il Discorso comunque ha voluto illustrare l'istituto, per così dire, dalle fondamenta, evitando ogni possibile equivoco o fraintendimento a fronte soprattutto del frequente decentramento o delega (anche mentale) della funzione giudiziaria ${ }^{40}$. Il Papa ha sottolineato espressamente la valenza del Vescovo come "giudice nato" della Chiesa particolare. Il concetto di giudice naturale (il giudice precostituito dalla legge) evoca la natura della potestà. Il titolare proprio della potestà giudiziaria, proprio per la necessaria concentrazione di potestà che caratterizza iure divino gli uffici capitali, non è solo il moderatore del suo tribunale (iudex unum et idem cum Vicario iudiciali), è anche il "ministro originario" del processo canonico. La competenza singolare del Vescovo-giudice prescinde evidentemente dalla costituzione di un proprio tribunale e assicura sempre (alle condizioni prescritte) un'estrema risorsa di giustizia matrimoniale locale, anche in circoscrizioni sprovviste di personale abilitato ${ }^{41}$. Il processus brevior, al di là della celerità $\mathrm{e}$

38 «Affinché sia finalmente tradotto in pratica l'insegnamento del Concilio Vaticano II in un ambito di grande importanza, si è stabilito di rendere evidente che il Vescovo stesso nella sua Chiesa, di cui è costituito pastore e capo, è per ciò stesso giudice tra i fedeli a lui affidati. [...] Ciò valga specialmente nel processo più breve, che viene stabilito per risolvere $\mathrm{i}$ casi di nullità più evidente» (III Criterio fondamentale, Proemio MIDI).

39 Cfr. ad es. C. M. Morán Bustos, El proceso "brevior" ante el Obispo diocesano, in M. E. Olmos Ortega (ed.), Procesos de nulidad matrimonial tras la reforma del Papa Francisco, Dykinson, Madrid 2016, 131-135; A. STANKIEWICZ, Alcune considerazioni intorno all'esercizio personale e vicario della potestà giudiziale con riferimento al processo matrimoniale breviore davanti al Vescovo, in AA. VV., Studi in onore di Carlo Gullo, III, LEV, Città del Vaticano 2017, 761-775 (spec. 768-771).

40 Larticolato prescrittivo è introdotto da una esplicita considerazione: «Da sempre il Vescovo diocesano è Iudex unum et idem cum Vicario iudiciali; ma poiché tale principio viene interpretato in maniera di fatto escludente l'esercizio personale del Vescovo diocesano, delegando quasi tutto ai Tribunali, stabilisco di seguito quanto ritengo determinante ed esclusivo nell'esercizio personale del Vescovo diocesano giudice» $\left(7^{\circ} \mathrm{cpv} .\right.$, Discorso). L'art. $22 \$ 2 \mathrm{DC}$, com’è noto, sconsigliava l'esercizio personale della potestà giudiziaria del Vescovo diocesano («Tuttavia è opportuno, a meno che speciali motivi lo richiedano, che egli non la eserciti personalmente»), circa i motivi del decentramento, cfr. anche J. Llobell, I processi matrimoniali nella Chiesa, EDUSC, Roma 2015, 134-136.

${ }^{41} \mathrm{Nel}$ caso soprattutto del ricorso ad un tribunale viciniore o limitrofo (can. $1673 \$ 2 \mathrm{MIDI}$ ). L'ipotesi del processus brevior è chiaramente distinta dalla facoltà di prevedere un giudice unico 
semplicità dell'accertamento, esprime la speciale cura e attenzione del Vescovo diocesano nei confronti dei suoi fedeli afflitti e sofferenti per il proprio stato matrimoniale. La proprietà dell'attribuzione evidenzia la qualificazione e sensibilità non professionale ma gerarchica e pastorale del giudice. Occorre sottolineare che la riserva della procedura alla mansione capitale non è solo simbolica o esemplare (l'autorevolezza di una pronuncia episcopale) ma garantista e funzionale all'amministrazione della giustizia secondo l'«unità cattolica nella fede e nella disciplina» ${ }^{42}$. Riteniamo che la personalità sia espressiva anche della pienezza dell'esplicazione del governo episcopale ${ }^{43}$, la facoltà normativa assicura infatti uno speciale e singolare supporto ratione munere al servizio giudiziario ${ }^{44}$. Ci sembra utile chiarire che il vincolo autoritativo (pur assumendo l'accezione ampia del ruolo episcopale delineata dal testo, infra $\$ 5.3)$ si riferisce solo alla potestà decisoria non alla fase introduttoria o preparatoria del giudizio ${ }^{45}$.

L'esclusività è un corollario o una derivazione della personalità: «La "personalità" che caratterizza il giudizio episcopale non concerne solo la monocraticità e la diretta conoscenza della causa ma l'infungibilità ed esclusività del potere del giudice in questo tipo di processo» ${ }^{46}$. Nel testo papale questa caratteristica è riportata pure come unicità $^{47}$. La notazione indica la non trasmissibilità della funzione decisoria, si preclude pertanto sia l'esercizio vicario che quello delegato del processus brevior. Un'insidia non troppo recondita che probabilmente il Pontefice ha avuto direttamente presente è stata quella di demandare l'attribuzione soprattutto nelle grandi circoscrizioni a un Ausiliare o ad un Vicario episcopale ${ }^{48}$. Il titolare proprio della potestà giudiziaria può

\footnotetext{
nel processo ordinario (can. $1673 \$ 4$, nulla esclude che il Vescovo possa assolvere personalmente anche questo ruolo).

${ }^{42}$ IV Criterio fondamentale, Proemio MIDI.

${ }^{43}$ Cfr. can. $381 \S 1$; LG 27.

44 «L'Ipse Episcopus iudex ha il senso in pratica di circoscrivere al suo munus pastorale l'uso della forma abbreviata» (M. DEL Pozzo, Il processo matrimoniale più breve..., cit., 65, l'espressione in corsivo è tratta dal III Criterio fondamentale, Proemio MIDI).

${ }^{45}$ Cfr. can. $135 \$ 4$.

${ }^{46}$ M. DEL Pozzo, Il processo matrimoniale più breve..., cit., 64.

${ }^{47} \mathrm{Cfr}$. $6^{\circ}$ cpv. e n. 1, Discorso. Si associa sempre comunque la personalità all'esclusività/unicità.

48 Fermo restando la unicità e peculiarità del regime della Sede romana, accanto alle pronunce personali del Santo Padre citate (supra nt. 24), non si può sottacere anche la sentenza, Romana, coram Augustino Card. Vallini (Vicario di Sua Santità Papa Francesco per la Diocesi di Roma), sentenza, 9-XII-2016, Prot. 17.830, in AA. Vv., Prassi e sfide dopo l'entrata in vigore del m.p. Mitis Iudex Dominus Iesus..., cit., 151-157. Non si conosce né il motivo della divergenza nello sbocco dei processi breviori né il numero delle cause inoltrate col rito abbreviato, ma, la posteriorità delle
} 
esercitarla in forma breviore ma non può mai disporne o trasferirla ad altri. Il ruolo capitale è infungibile quoad munus e la riserva è assoluta. La preclusione della sostituzione non risponde solo ad un motivo disciplinare ma alla ratio ecclesiologica e teleologica che ispira l'istituto. Le condizioni previste ad validitatem per la titolarità del giudizio d'altronde precisano perentoriamente che deve trattarsi del «capo di una comunità diocesana di fedeli» (infra $\$ 4.2$ ).

\subsection{L'individuazione e la giustificazione del Giudice Vescovo}

La precisazione ermeneutica più netta e chiara concerne l'individuazione del giudice del processus brevior: «Nel processo breviore sono richieste, ad validitatem, due condizioni inscindibili: l'episcopato e l'essere capo di una comunità diocesana di fedeli (cfr. can. $381 \$ 2$ ). Se manca una delle due condizioni il processo breviore non può aver luogo. L'istanza deve essere giudicata con il processo ordinario» (n. 3). Non si tratta tanto di una nuova statuizione quanto della delucidazione del contenuto della formula legislativa adoperata (Vescovo diocesano). La spiegazione elimina così dubbi o perplessità circa l'esatta determinazione del titolare della forma processuale ${ }^{49}$. Il requisito sacramentale e quello potestativo stabiliti devono necessariamente concorrere ad integrare la fattispecie. Il presupposto soggettivo è inoltre tassativo per accedere al processo breviore, l'eventuale inibizione non preclude peraltro il normale esame giudiziario (tramite il processo ordinario $)^{50}$. La formula irritante determina la nullità della sentenza indebitamente emessa da un giudice che non riunisca le condizioni previste ${ }^{51}$.

La riserva del processus brevior al carattere episcopale implica l'intrinseco collegamento dell'istituto con la successione apostolica e con il nucleo della comunio-

pronunce papali, può indicare, oltre ad una contestualità di attribuzioni (cfr. anche art. $32 \$ 1$ Giovanni PAOLO II, cost. ap. Ecclesia in Urbe, 1-I-1998 [«Il Cardinale Vicario, in virtù della potestà ordinaria vicaria che esercita in nome del Sommo Pontefice, è giudice ordinario (cfr. can. 1419 CIC) della Diocesi di Roma e Moderatore dei Tribunali»]), una revisione o correzione dell'orientamento precedentemente assunto.

49 Al riguardo formulavamo l'auspicio di un intervento chiarificatore: «Una questione pregiudiziale fondamentale concerne l'individuazione del soggetto legittimato a pronunciare la sentenza tramite il processus brevior. [...] Precisiamo subito che al riguardo sarebbe auspicabile e più rassicurante un chiarimento autoritativo del dettato normativo» (M. DEL PozZO, Il processo matrimoniale più breve..., cit., 59-60).

50 La fungibilità del rito per il fedele è imprescindibilmente subordinata alla sussistenza delle condizioni, oltre al previo controllo potestativo discrezionale (normalmente affidato al Vicario giudiziale).

${ }^{51}$ Cfr. can. 10. 
ne ecclesiale. Il chiarimento ha comportato anche una giustificazione ecclesiologica e dottrinale della scelta compiuta. La specifica facoltà giudiziaria è stata ricondotta alla pienezza di potestà derivante solo dalla consacrazione episcopale ${ }^{52}$. Il chiarimento esige quindi una doverosa ritrattazione dell'opinione precedentemente espressa circa il vincolo non strettamente sacramentale dell'attribuzione $^{53}$. Motivi razional-sistematici (la natura della potestà giudiziaria e la distinzione dell'aspetto giurisdizionale da quello carismatico) e garantistici (l'assicurazione di uno strumento di giustizia a fedeli di circoscrizioni più disagiate) inducevano a preferire un'interpretazione ampia ed estensiva della locuzione tipologica adoperata (Episcopus diocesanus). La soluzione adottata comunque è sicuramente più fedele al dato letterale e alla mens legislativa. La delucidazione è in linea con la preoccupazione dottrinale e disciplinare nell'amministrazione della giustizia palesata dal Santo Padre ${ }^{54}$.

Per quanto riguarda il secondo elemento («l'essere capo di una comunità diocesana di fedeli») l'identificazione del requisito è leggermente più complessa. Il chiarimento richiama per relationem, come sembra logico e ragionevole, anche i soggetti equiparati al Vescovo diocesano che presiedono strutture assimilate alla diocesi ${ }^{55}$. Chiaramente se però il Pastore difettasse della consacrazione episcopale viene meno il presupposto fondante della forma processuale $^{56}$. Il codice non menziona le missiones sui iuris che abitualmente non sono rette da un Vescovo. Il concetto di 'comunità diocesana di fedeli' suppone il riferimento ad una Chiesa particolare ovvero ad una struttura giurisdizionale necessaria. Mentre ci sembra si possono escludere altre circoscrizioni complementari, dubbi possono sorgere sull'ammissibilità del processo più breve negli Ordinariati militari e negli Ordinariati personali (sempre che l'Ordinario sia un Vescovo ${ }^{57}$ ). Tali enti si occupano infatti della pastorale ordinaria e familiare dei fedeli con un carattere comunitario e gerarchico. Gli Ordinariati militari sono espressamente assimilati alle diocesi nella cost. Spi-

${ }^{52}$ Cfr. n. 4, Discorso. La puntualizzazione non altera il carattere giurisdizionale dell'attribuzione e la centralizzazione normativa del Romano Pontefice.

53 Cfr. M. DEL Pozzo, Il processo matrimoniale più breve..., cit., 59-64.

${ }^{54}$ Cfr. IV Criterio fondmentale, Proemio MIDI.

${ }_{55}$ Cfr. il combinato disposto dei cann. $381 \$ 2$ e 368 (si tratta della prelatura e abbazia territoriale, della prefettura e vicariato apostolici e dell'amministrazione apostolica stabilmente eretta).

${ }^{56}$ Ciò si verificherà abitualmente nelle abbazie territoriali e talora nelle prefetture apostoliche (cfr. PAOLO VI, m. p. Catholica Ecclesia, 23-X-1976, n. 4, AAS 68 [1976] 696; Annuario Pontificio 2017, 1055-1059).

57 Circostanza abbastanza frequente negli Ordinariati militari e più rara in quelli personali. 
ritalium militum curae $^{58}$. La qualificazione letterale (che risente delle categorie dell'epoca) non è tuttavia sufficiente a determinarne la natura ecclesiologica. L'approfondimento concettuale successivo ha chiarito che si tratta piuttosto di entità cumulative e di supporto in ragione della specificazione logistica e professionale ${ }^{59}$. Gli ordinariati castrensi non costituiscono quindi comunità pastorali essenziali e necessarie rapportabili alle diocesi. Non trattandosi di Chiese particolari (lato sensu intese) riteniamo perciò che l'Ordinario non possa essere equiparato al Vescovo diocesano. Per gli Ordinariati personali viceversa la generalità e globalità del servizio e, soprattutto, l'esclusività dell'appartenenza, per quanto discussa e complessa, deporrebbero per una ragionevole assimilazione alle comunità diocesana ${ }^{60}$. Non si rinviene pertanto un ostacolo istituzionale all'ammissibilità del processus brevior. Il possibile e frequente difetto del carattere episcopale comunque circoscrive l'ipotesi ${ }^{61}$.

\subsection{La responsabilità del Vescovo-Giudice nel 'processus brevior'}

Il Discorso pontificio contiene un forte richiamo alla responsabilità del Vescovo. Come accennato, un intento dichiarato di Papa Francesco è di sensibilizzare il corpo episcopale alla necessità dell'attuazione della riforma e del processus brevior in particolare in maniera dimostrativa ed esemplare (ma non per questo strumentale o populistica) della sollecitudine e attenzione per i fedeli in difficoltà. Si può dunque considerare una puntualizzazione importante e illuminante l'obbligatorietà della concessione della possibilità di introdurre la procedura abbreviata: «Il processo breviore non è un'opzione che il Vescovo dioce-

${ }^{58}$ Cfr. Giovanni Paolo II, cost. ap. Spiritalium militum curae, 24-IV-1986, n. I $\$ 1$ («Gli Ordinariati militari, che si possono chiamare anche castrensi e che vengono giuridicamente assimilati alle diocesi,...»), n. II $\$ 1$ («All’Ordinariato militare è preposto, come proprio, un Ordinario normalmente insignito della dignità episcopale, il quale gode tutti i diritti ed è tenuto agli obblighi dei vescovi diocesani, a meno che non consti diversamente dalla natura delle cose o dagli statuti particolari»).

59 «Por su parte, la calificación de la potestad del ordinario castrense como cumulativa impide considerarla exenta, separada o independiente de los obispos diocesanos» (A. VIANA, «Ordinariato militar», in J. Otaduy - A. Viana - J. Sedano [eds.], Diccionario General de Derecho Canónico, V, Thomson Reuters Aranzadi, Cizur Menor [Navarra] 2012, 810, ivi anche ampia bibliografia).

${ }^{60}$ Cfr. Benedetto XVI, cost. ap. Anglicanorum coetibus, 4-XI-2009, art. I $₫ 3$ («Ciascun Ordinariato ipso iure gode di personalità giuridica pubblica; è giuridicamente assimilato ad una diocesi»). La figura degli Ordinariati personali necessita probabilmente di un ulteriore inquadramento sistematico e approfondimento critico. Ogni valutazione deve comunque tener conto delle specifiche Norme complementari previste.

${ }^{61}$ Cfr. Annuario pontificio 2017, 1028 (2 dei 3 attuali Ordinari personali non sono Vescovi). 
sano può scegliere ma è un obbligo che gli proviene dalla sua consacrazione $\mathrm{e}$ dalla missio ricevuta» (n. 5). La giustificazione e il presupposto della forma processuale (consacrazione episcopale e preposizione gerarchica) fungono quindi da titolo di esigenza della specifica prestazione giudiziaria. L'ampliamento (qualitativo) dell'offerta di giustizia manifesta concretamente l'istanza di condivisione e collaborazione tra primato e collegialità nello spirito sinodale ${ }^{62}$. La spinta del popolo e dei Padri a venire incontro ai fedeli oppressi «dalle tenebre del dubbio», si è concretizzata infatti in un atto normativo del Romano Pontefice che impegna personalmente e istituzionalmente il titolare proprio della potestà e il garante locale dell'unità e della comunione. Vedremo in seguito (infra $\$ 5$ ) come l'intimazione possa non trasformarsi in un'ingiunzione immediata, la comprensione per eventuali difficoltà o lentezze non sminuisce comunque l'essenza vincolante del dovere. Il riscontro e la praticabilità della forma sono rimessi alla discrezionalità del Vescovo quanto al quomodo e al quando ma non quanto all'an. L'accessibilità dello strumento processuale semplificato (ove si diano gli estremi legali) non è nella scelta o disponibilità organizzatoria del Vescovo ${ }^{63}$.

Il discorso circa l'infungibilità, almeno in linea di principio, della modalità del processus brevior deve essere distinto da quello concernente il livello di coinvolgimento del Vescovo che esamineremo in seguito (infra $\$ 5.3$ ). Al di là della competenza o, piuttosto, supervisione sull'intero processo, è un principio fondamentale, espressamente ribadito e chiarificato, che la responsabilità del Vescovo non è solo garantistico-formale o "notarile" ma essenziale e sostanziale nell'economia del giudizio. Il ruolo del Vescovo, così come incisivamente delineato («la figura del Vescovo-diocesano-giudice è l'architrave, il principio costitutivo e l'elemento discriminante dell'intero processo breviore»), non indica certo una sorta di apparenza o "copertura" autoritativa della pronuncia, ha un valore selettivo e qualificante della procedura. Integra la $\mathrm{ra}$ tio del processus brevior soprattutto la riconduzione della decisione al libero convincimento e alla certezza morale (ex actis et probatis) del Giudice-Vescovo. La centralità della coscienza del Presule assume allora una valenza determinante e dirimente ${ }^{64}$. Ridurre il Vescovo a «mero firmatario della sentenza»

${ }^{62}$ È abbastanza significativo l'incipit delle Regole procedurali annesse al MIDI [= RP MIDI].

${ }_{63}$ A rigore potrebbero mancare, almeno temporaneamente, le forze per costituire un tribunale, ma mai la figura e la persona del Vescovo giudice.

${ }^{64}$ Cfr. supra nt. 21. 
equivale a snaturare e stravolgere il principio del giusto processo. L'equivoco concettuale o il cedimento operativo, pur supposto palesemente o surrettiziamente da alcuni, è stato perciò esplicitamente censurato e riprovato dal Papa in maniera chiara e univoca. La puntualizzazione, oltre che direttiva, è anche risolutiva e interpretativa. A parte la responsabilità esterna o organizzativa, esiste un'ancor più rilevante implicazione interna nel merito della causa.

\subsection{La potestà circa l'appello del Decano della Rota}

Un'ulteriore precisazione categorica concerne la natura della potestas decidendi preliminare attribuita al Decano (in caso di appello alla Rota Romana ex can. 1687 \$3). Il Papa chiarisce che l'attribuzione è «nuova e dunque costitutiva», deriva dalla statuizione del can. $1687 \$ 4$. Si tratta invero di una giustificazione della prescrizione normativa più che di una risoluzione ermeneuti$\mathrm{Ca}^{65}$. La puntualizzazione deriva probabilmente dalla fissazione del necessario carattere episcopale del primo Giudice ${ }^{66}$. L'esigenza sacramentale riguarda solo la garanzia pastorale e gerarchica del giudizio in sede locale ma non vincola la strutturazione dell'amministrazione della giustizia nel governo centrale. La disponibilità delle caratteristiche del giudice d'appello rientra pacificamente nella discrezionalità normativa del Legislatore ${ }^{67}$. La riforma, com'è noto, ha espressamente sancito l'opportunità della conservazione del collegamento delle Chiese particolari con la Sede Apostolica nella facoltà d'appello ${ }^{68}$. L'affermazione comunque salvaguarda l'organicità e coerenza dell'intervento pontificio e sottolinea la specificità decisoria dell'esame previo ${ }^{69}$.

Il punto in esame ribadisce il contenuto della prescrizione codiciale quanto all'esame previo. Le espressioni usate («rigetto o ammissione dell'appello»), per quanto non riteniamo che bisogna attribuire un valore ulteriore rispetto al senso del chiarimento, non sembrerebbero legittimare un'equipa-

${ }^{65}$ Non sorgevano eccessivi dubbi sulla legittimazione ex lege del Decano della Rota, benché in dottrina fosse stata espressa qualche riserva sulla misura disposta.

${ }^{66}$ Com'è noto, il Decano della Rota, può non essere Vescovo e abitualmente non lo è stato (cfr. anche art. 1 Normae Romanae Rotae Tribunalis).

${ }^{67}$ Non si tratta di una deroga o eccezione ai principi del sistema processuale. Ł̀ stato espressamente chiarito che la potestà giudicante in secondo grado deriva dalla potestà primaziale (J. LLOBELL, I processi matrimoniali..., cit., 157).

${ }^{68}$ Cfr. VII Criterio fondamentale, Proemio MIDI.

${ }^{69}$ Cfr. M. DEL Pozzo, L'appello nel 'processus matrimonialis brevior', Archivio Giuridico 237/3-4 (2017) 485-535 (spec. 495-512). 
razione del riscontro negativo (reiectio in limine) all'eventualità della conferma, come pure sostenuto da autorevole dottrina ${ }^{70}$.

Il capoverso successivo non è di semplice e immediata comprensione ( «In conclusione, vorrei ribadire con chiarezza che ciò avviene senza chiedere il permesso o l'autorizzazione ad altra Istituzione oppure alla Segnatura Apostolica»). Per la collocazione sistematica si deve supporre che la proposizione sia collegata con il chiarimento precedente ${ }^{71}$. Per il giudizio del Decano si esclude il bisogno di qualsivoglia nulla osta o una commissione da parte della Segnatura (ricostruzione peraltro abbastanza scontata). Un'altra più remota ipotesi interpretativa è sottrarre il provvedimento di rigetto a un'eventuale impugnazione (equivarrebbe alla qualifica expeditissime) ${ }^{72}$. Limpostazione reiterativa e negativa della puntualizzazione ad ogni modo esclude patentemente un intento innovativo e rende probabilmente superflue eccessive disquisizioni.

\section{LE INDICAZIONI SOLLECITATORIE E DIRETTIVE}

Qualifichiamo come "indicazioni sollecitatorie" le istruzioni fornite dal Santo Padre che non presentano una valenza interpretativa stringente o un carattere direttamente vincolante ${ }^{73}$. L'incidenza applicativa meno immediata non significa però che tali richiami difettino di fermezza e chiarezza o che siano solo tendenziali e meramente propositivi. Le avvertenze orientative e di principio in un certo senso sono ancor più fortemente identificatrici e rivelatrici dello spirito della normativa e dei desideri del Supremo Legislatore. L'esecuzione dei chiarimenti richiede tuttavia il confronto con la particolarità delle situazioni locali e l'integrazione con la decisione prudenziale dei Vescovi. Con realismo e buon senso il Papa non ha voluto imporre una scelta im-

${ }^{70}$ Cfr. G. P. MONTINI, «Si appellatio mere dilatoria evidenter appareat» (cann. $1680 \$ 2$ e $1687 \$ 4$ MIDI): alcune considerazioni, Periodica 105 (2016) 690-691. Anche SUPREMo Tribunale DELLA Segnatura Apostolica, Lettera circolare "Inter munera" sullo stato e l'attività dei Tribunali, 30VII-2016, Prot. n. 51712/16 VT, a proposito delle Causae nullitatis matrimonii per processum breviorem coram Episcopo pertractatae in seconda istanza indica nella relativa modulistica di relazione: «42. Decreta confirmatoria (seu appellationes in limine reiectae)».

${ }^{71}$ L'incipit «In conclusione» potrebbe però dare un intento riepilogativo dei chiarimenti (che però rimarrebbe oscuro).

72 Cfr. M. DEL Pozzo, L'appello nel 'processus matrimonialis brevior', cit., 510-512 (3.1.4. I rimedi contro il decreto di rigetto). Il parlare di 'permesso' o 'autorizzazione' paiono contrastare una simile deduzione.

${ }^{73}$ L'esecuzione pratica pare rimessa all'opportuna valutazione dei singoli Vescovi. 
perativa ma rinviare alla diligenza e laboriosità dei singoli Pastori la tempistica e il modo più conveniente per assicurare l'effettività e rispondenza del processus brevior. La valenza prescrittiva di questi profili dell'intervento non si può evidentemente trasformare in un espediente per rinviare o eludere sine die l'applicazione della voluntas Legislatoris.

\subsection{L'attuazione della modalità processuale}

Abbiamo già chiarito che la responsabilità del Vescovo sta nel garantire l'accessibilità e proficuità della nuova forma processuale (supra $\$ 4.3$ ): l'attuazione del processus brevior non è opzionale o facoltativa ma doverosa e obbligatoria. A fronte della difficoltà strutturale o della possibile impreparazione tecnica e professionale di alcuni Presuli; si prende in considerazione l'eventualità di ritardi o rinvii ${ }^{74}$. Il quam primum indicato non può paradossalmente legittimare una lentezza o indeterminatezza operativa. La sollecitazione concerne allora la premura e tempestività nella dotazione organica e nel riscontro alle esigenze dei fedeli. La mancata disponibilità attuale all'applicazione della forma abbreviata costituisce una motivata eccezione e una restrizione funzionale (più che una deroga o un privilegio personale ${ }^{75}$ ). La regola e la prassi dovrebbe essere la possibilità immediata e generalizzata di accesso al processo più breve. L'auspicio è dunque che ubique sia possibile celebrare un processo davanti al Vescovo. È interessante considerare che la momentanea inattuazione dello strumento semplificato per un motivo soggettivo comporta l'adeguata e, in un certo senso, rafforzata vigilanza per la celerità del processo ordinario. Il bene dei fedeli in pratica non può essere oltremodo compromesso dalle scelte del Pastore ${ }^{76}$.

La misericordia e la sensibilità pastorale spinge dunque a impegnarsi in questo fronte processuale. La forte spinta all'accessibilità della procedura non si trasforma evidentemente di un uso indebito, generalizzato e arrendevole del

${ }^{74}$ Cfr. G. BeLfiore, I processi di nullità matrimoniale..., cit., 163-164, che ha rilevato la mancanza di un adeguato regime transitorio nella riforma.

${ }^{75}$ Non avrebbe senso legittimare una contestazione o resistenza concettuale all'impegno e alla formazione canonistica e umana matrimoniale.

${ }^{76}$ L'adozione (o la mancata attuazione) del processus brevior è comunque un incentivo e uno stimolo a velocizzare il processo ordinario, che sarà la via normale e abituale. Lo stesso comparativo (brevior) suppone che il processus ordinarius sia brevis, cfr. M. DEL POZZO, Il processo matrimoniale più breve..., cit., 30-31. 
giudizio personale del Vescovo ${ }^{77}$. L'eventualità dell'esenzione dall'immediata attuazione («nel caso poi non si ritenesse pronto nel presente ad attuarlo») indica una delicata forma di comprensione e rispetto nei confronti delle insufficienze o insicurezze dei confratelli nell'episcopato ${ }^{78}$. L'accondiscendenza o, piuttosto, la tolleranza ammessa, ad ogni modo, dovrebbe implicare una semplice moratoria o proroga nell'attuazione ${ }^{79}$. L'onere formativo diretto e indiretto del Vescovo ha insomma un contenuto precettivo ineludibile. Le carenze o i limiti canonistici sono evidentemente colmabili e superabili col tempo, lo studio e l'applicazione $^{80}$. Se «la figura del Vescovo-diocesano-giudice è l'architrave, il principio costitutivo e l'elemento discriminante dell'intero processo breviore», la qualità del processo deriva dalla preparazione personale, dalla dedizione, prudenza e riflessione del Vescovo. Alla formazione e aggiornamento individuale si associa anche l'obbligo di provvedere all'istruzione e cultura di validi collaboratori ${ }^{81}$.

77 Diversi autori hanno sottolineato la straordinarietà o, quantomeno l'estrema cautela, nel ricorso al processus brevior: «In another respect, however, a careful examination of the institute reveals that its use is designed to be exceptional and even somewhat rare in pratice» $(W . \mathrm{L}$. DANIEL, The Abbreviated Matrimonial Process before the Bishop in Cases of "Manifest Nullity" of Marriage, The Jurist 75 [2015] 590); « $\mathrm{E}$ inutile che mi soffermi su alcuni punti ormai condivisi dalla dottrina o comunque sufficientemente suffragati dal testo di legge, ossia i seguenti: $1^{\circ}$. La natura straordinaria o eccezionale del processus brevior, intesa nel senso di una sua occorrenza statisticamente molto bassa rispetto al numero delle cause di nullità portate dinanzi ai tribunali ecclesiastici; questo dato pare confermato dalle prime relazioni sullo stato e l'attività dei tribunali nel 2016, che giungono in Segnatura Apostolica in questo scorcio di anno, e contribuisce a dimensionare la forse eccessiva letteratura su questo processo; [...]» (G. P. MonTINI, Gli elementi pregiudiziali del processus brevior: consenso delle parti e chiara evidenza di nullità, in Prassi e sfide dopo l'entrata in vigore del m.p. Mitis Iudex Dominus Iesus..., cit., 47-48); «El proceso breve, tal como ha sido configurado por el legislador, aparece como un proceso extraordinario y excepcional, y como tal debería ser aplicado en la praxis forense canónica» (C. M. MORÁN BUSTOS, El proceso "brevior"..., cit., 175); «Por el otro lado, se trata de un proceso especial, extraordinario, que contiene significativas modificaciones respecto al proceso contencioso-ordinario de nulidad matrimonial, cuya normativa actúa como supletoria» (C. PEÑa GARCía, La reforma de los procesos canónicos de nulidad matrimonial: el motu proprio «Mitis Iudex Dominus Iesus», Estudios Eclesiásticos 90 [2015] 663).

${ }^{78}$ Cosciente della complessità del giudicare, la valutazione riguarda solo l'incapacità o incompetenza tecnica del Giudice-Vescovo non le perplessità o riserve circa il provvedimento legislativo che rientra nella potestà del Supremo Legislatore e richiede l'obbedienza dei destinatari (cfr. can. $12 \$ 1)$. Non si configura certo una sorta di obiezione di coscienza.

79 Per quanto sembri lodevole un'adeguazione e un aggiornamento, Vescovi ormai prossimi alla scadenza o in condizioni molto precarie potrebbero di fatto permanentemente non ritenersi in grado di assolvere correttamente tale funzione giudicante personale e delicata.

80 A prescindere dalla previsione di una minima formazione canonistica nel curriculum di qualunque sacerdote, non bisogna sottolineare troppo il deficit di preparazione giuridico-matrimoniale dei Vescovi, cui si può ovviare con lo studio, l'applicazione e il consiglio.

${ }^{81}$ Cfr. art. $8 \$ 1 R P M I D I$; nonché Congregazione PER L'EduCaZione CaTTOLICA, istr. Gli studi di Diritto Canonico alla luce della riforma del processo matrimoniale, 29-IV-2018. 
L'abilitazione delle altre figure (istruttore, assessore, notaio, difensore del vincolo) rientrano nelle urgenze della pastorale matrimoniale, avendo presente che la professionalità è un dato che non si scopre o improvvisa ma che si deve forgiare pazientemente e perfezionare ${ }^{82}$.

\subsection{La preoccupazione "globale" del Vescovo}

All'an si aggiunge il quomodo, il Santo Padre ha voluto richiamare espressamente la serietà e responsabilità del giudizio episcopale. Il Pontefice ha sottolineato perciò il coinvolgimento personale del Vescovo diocesano: «Egli è competente esclusivo nelle tre fasi del processo breviore» (introduttoria, istruttoria, decisoria). Il punto in questione (n. 5) è uno dei passaggi più delicati e difficili del Discorso. Riteniamo che il Papa abbia voluto mettere in guardia soprattutto contro un esercizio distaccato e "notariale" della funzione del Vescovo giudice (non può limitarsi a sovrintendere o ratificare l'operato dei suoi ministri). La semplice garanzia formale ed esterna evidentemente tradirebbe l'identità e la mansione del giudice. Anche per l'impostazione e il contenuto dell'intervento, non ci sembra peraltro che i chiarimenti o, in questo caso, le indicazioni o puntualizzazioni abbiano il senso di mutare o innovare l'assetto normativo dei Motu Proprio ${ }^{83}$. Il Legislatore ha cercato solo di esplicitare operativamente la disciplina vigente. La struttura tipica del processus brevior è data dalla competenza decisoria (personale ed esclusiva) del Vescovo, senza però la necessità di un'immediatezza e concentrazione preparatoria e istruttoria. L'intervento finale e risolutorio del giudiceVescovo non comporta però un'estraneità o incompetenza rispetto allo sviluppo del processo. La natura del Giudice anzi configura e condiziona l'intera procedura. Considerata anche l'articolazione del Discorso, conviene esaminare brevemente il ruolo del Vescovo diocesano nelle tre fasi o momenti del giudizio.

Il Discorso recepisce le indicazioni del Sussidio applicativo circa l'indiriz$z 0$ dell'istanz $a^{84}$. L'invocazione del ministero giudicante del Vescovo è diretta al Pastore stesso e non al relativo tribunale. Lipotesi si riferisce all'introduzione

${ }^{82} \mathrm{Al}$ Vescovo diocesano comunque non viene richiesto certo di essere un giudice professionale, basta che svolga con serietà e prudenza il suo compito.

${ }^{83}$ Ribadiamo che il Santo Padre non ha proceduto a correzioni o integrazioni del dettato legale.

${ }^{84}$ Il Sussidio applicativo distingue la domanda dal libello: «La domanda va presentata al Vescovo e/o al Vicario giudiziale diocesano. Il libello, presentato al Vicario giudiziale diocesano, deve esporre i fatti, indicare le prove ed esibire in allegato i documenti su cui si fonda la domanda» (Quadro riassuntivo, p. 37). Nella p. 19 comunque si precisa espressamente e ripetutamente che la domanda e il libello vanno indirizzati sempre al Vescovo. 
della causa esplicitamente a mezzo del processus brevior, ciò non esclude il vaglio o l'esame della domanda da parte del Vicario giudiziale o del soggetto deputato ${ }^{85}$. La normativa prevede anche la possibilità della "conversione" della domanda di giustizia dal processo ordinario a quello breviore quando se ne ravvisino gli estremi e sussistano le condizioni su iniziativa dell'Officiale. In tal caso ovviamente solo successivamente l'istanza sarà rivolta al Vescovo. La "esclusiva competenza" può avere anche il senso di deferire ogni eventuale ricorso o contestazione nella fase introduttoria (o istruttoria) al giudizio del Vescovo, la questione richiede però una spiegazione e approfondimento più attento ${ }^{86}$.

Per quanto concerne l'istruttoria il "giudice naturale" può sempre evidentemente svolgerla in prima persona ${ }^{87}$. Non si richiede tuttavia che il Vescovo presieda alla raccolta delle prove e normalmente è abbastanza irrealistico che lo faccia ${ }^{88}$. Il passo del Discorso citato non tralascia peraltro l'ausilio delle figure previste dalla normativa (istruttore, assessore, difensore del vincolo). La competenza di specialisti o collaboratori («sempre coadiuvato»), ancorché circostanziata e individuata, è quindi parte integrante e imprescindibile del modello processuale delineato. Se l'istruttoria fosse istituzionalmente demandata al Vescovo non si comprenderebbe il menzionato ricorso al tribunale viciniore in assenza di canonisti nella propria comunità diocesana ${ }^{89}$. Il coinvolgimento del Vescovo pure nel momento probatorio implica comunque l'attenta supervisione e controllo circa il materiale raccolto, la possibile soluzione di contrasti e l'eventuale richiesta di un supplemento istruttorio ${ }^{90}$. Il Santo Padre ha voluto ribadire l'aspirazione della conclusione della fase istruttoria in una sola sessione. Il contenimento temporale però è tendenziale e orientativo, in linea con la semplicità e rapidità dell'accertamento, ma non è tassativo ("abitualmente") ${ }^{91}$.

${ }^{85}$ Il can. $1676 \S 2$ e l'art. 15 RP MIDI non stabiliscono un automatismo nell'ammissione ma una valutazione discrezionale a cura del Vicario giudiziale.

${ }^{86}$ Cfr. M. DEL Pozzo, Considerazioni sui ricorsi della fase introduttiva del giudizio matrimoniale nell'impianto del m. p. "Mitis iudex", Stato, Chiese e pluralismo confessionale, Rivista telematica (www.statoechiese.it) n. 34 (2016) 1-24.

87 Nel Discorso del 12 marzo 2016 richiamato, è stato esemplificativamente proposto un caso in cui il Vescovo, opportunamente coadiuvato, assume la direzione dell'indagine.

${ }^{88}$ Il tenore del can. $1687 \$ 1$ è abbastanza esplicito («Ricevuti gli atti, il Vescovo diocesano, consultatosi con l'istruttore e l'assessore, ...»).

${ }^{89}$ Cfr. anche Sussidio applicativo, p. 19, circa il modo di garantire almeno l'ausilio e il supporto di un canonista nel giudizio del Vescovo.

90 L'esigenza di un'istruttoria più accurata e dettagliata difficilmente potrebbe non condurre all'emissione del decreto di remissione all'esame ordinario.

91 Cfr. anche P. BIANCHI, Lo svolgimento del processo breve: la fase istruttoria e di discussione della causa, in REDAZIONE DI QUADERNI DI DIRITTO ECCLESIALE (ed.), La riforma dei processi matrimoniali di 
Per quel che riguarda la soluzione del processo, l'indicazione pontificia è perentoria: «la decisione da pronunciare coram Domino, è sempre e solo del Vescovo diocesano». L'avvertenza puntualizza che la pronuncia deve essere assunta in coscienza (coram Domino) ed è di esclusiva pertinenza del Vescovodiocesano-giudice, senza che possano ammettersi eccezioni. Nel caso della sentenza affermativa la certezza morale sarà raggiunta ex actis e probatis secondo l'intima convinzione dal Vescovo ${ }^{92}$. Il Giudice (in questo caso il Vescovo) non può mai limitarsi a recepire la persuasione maturata dai coniugi ${ }^{93}$. Nel punto successivo si addita come un deplorevole cedimento l'atteggiamento burocratico o elusivo del Vescovo («snaturare e ridurre la figura del Vescovo padre, capo e giudice dei suoi fedeli a mero firmatario della sentenza»). Sta di fatto che l'adesione all'altrui convincimento, fermo restando magari la buona fede, integra un inganno in un atto pubblico. Ciò non significa che non possa essere demandata ad altri la redazione della decisione, sulla base però degli elementi assunti e della convinzione maturata dal Vescovo ${ }^{94}$. La motivazione è la esplicitazione o giustificazione dell'effettivo giudizio episcopale.

Il Papa in pratica richiama il Vescovo giudicante all'integralità e compiutezza della sua missione giudiziaria, senza schermi o rinunce. In linea con l'impianto della riforma, il Santo Padre sembra stimolarne la piena e sostanziale applicazione del disposto, con le aperture e la flessibilità che consente. La "gestione" del processus brevior (sia nell'organizzazione che nella concreta conduzione) manifesta d'altronde la preoccupazione e sollecitudine per gli istanti ${ }^{95}$.

papa Francesco. Una guida per tutti, Àncora, Milano 2016, 67-90 (spec. 82-83); IDEM, Il processus brevior coram Episcopo. Le prime esperienze di un tribunale locale, in Studi in onore di Carlo Gullo, III, LEV, Città del Vaticano, 799-826 (spec. 821-823). L'A. propendeva per una considerazione della sessione più ampia della semplice udienza.

92 Cfr. J. Llobell, La certezza morale nel processo canonico, Il Diritto Ecclesiastico 109/1 (1998) 758802; J. Yunga Ndosimau, Les actes judiciaires dans la structure de la certitude morale à la lumiere de l'art. 247 de la Dignitas connubii, Médiaspaul, Kinshasa 2011; A. STANKIEWICZ, La certezza morale e la motivazione della sentenza, in H. FrANCESCHI - J. LLOBELL - M. Á. ORTIZ (eds.), La nullità del matrimonio: temi processuali e sostantivi in occasione della «Dignitas Connubii», EDUSC, Roma 2005, 231-245.

93 Cfr. M. DEL Pozzo, La centralità della coscienza nella verità del matrimonio, 375-378.

94 «La sentenza deve essere firmata personalmente dal Vescovo (ma può essere estesa, ad esempio, dall'Assessore o dallo stesso istruttore)» (p. 40, Sussidio applicativo MIDI). Cfr. anche art. $48 \$ 2$ Lex propria Signaturae Apostolicae 2008.

95 La prontezza della risposta alla domanda di giustizia dei fedeli è un'evidente dimostrazione di ascolto e attenzione. 


\subsection{Il tribunale competente e l'organizzazione giudiziaria}

Un'ulteriore indicazione ricavabile dal testo concerne l'individuazione del tribunale competente. La dimensione propria della giustizia matrimoniale episcopale sembra decisamente quella diocesana: «Affidare l'intero processo breviore al tribunale interdiocesano (sia del viciniore che di più diocesi) porterebbe a snaturare e ridurre la figura del Vescovo padre, capo e giudice dei suoi fedeli a mero firmatario della sentenza» (n. 6). Il punto si collega anche all'invocazione della prossimità come valore permanente della riforma processua$\mathrm{l}^{96}$. È stato rilevato come l'indubbio orientamento programmatico della riforma verso il ritorno al tribunale diocesano si è dovuto confrontare e, in parte, "ridimensionare" (sicuramente in Italia, ma anche in altri contesti geografici) con la pregressa situazione organizzativa e le notorie carenze di personale, di mezzi e di strutture, imponendo spesso, pragmaticamente e con buon senso, soluzioni, almeno temporanee, di mantenimento del servizio esistente $^{97}$. La possibile concorrenza o contestualità tra il livello diocesano e interdiocesano sembrava già inclinare a favore della giurisdizione più vicina $\mathrm{e}$ immediata per il processus brevior ${ }^{98}$. L'intervento pontificio suffraga decisamente questa istanza, mirando a scongiurare una visione funzionariale e distaccata dell'operato del Vescovo.

I desiderata e gli auspici del Legislatore sono chiari, la soluzione però non è draconiana e imperativa, è rimessa alla valutazione e considerazione dei singoli Vescovi. Il testo del Discorso infatti non destituisce di fondamento e legittimità il ricorso al tribunale interdiocesano o viciniore. La stessa regolamentazione legale nel processus brevior sembra consentire e supporre l'eventualità dell'affidamento dell'introduzione e dell'istruttoria al tribunale interdiocesano ${ }^{99}$. L'eventualità del supporto e il soccorso di un

${ }^{96}$ Cfr. n. 8 Discorso, infra $\$ 6$.

97 G. BeLfiore, I processi di nullità matrimoniale..., cit., 137-171, con un po' di amarezza e disappunto lamenta un cedimento operativo rispetto all'impianto organizzativo prospettato dai Motu proprio.

98 Cfr. M. DEL Pozzo, I titoli di competenza e la "concorrenza materiale" alla luce del m. p. Mitis iudex Dominus Iesus, Ius Ecclesiae 28 (2016) 457-478 (spec. 467-473). Il Sussidio applicativo MIDI già indicava la competenza del Vicario giudiziale diocesano (18-19).

99 «Il Vicario giudiziale può designare se stesso come istruttore; però per quanto sia possibile nomini un istruttore dalla diocesi di origine della causa» (art. $16 R P M I D I)$; «Se la causa viene istruita presso un tribunale interdiocesano, il Vescovo che deve pronunziare la sentenza è quello del luogo in base al quale si stabilisce la competenza a mente del can. 1672. Se poi siano più di uno, si osservi per quanto possibile il principio della prossimità tra le parti e il giudice» (art. $19 R P M I D I)$. 
Vescovo viciniore d'altronde è richiamato pure dalle parole del $\mathrm{Papa}^{100}$. Il forte auspicio che si giunga in tempi ragionevolmente rapidi a una amministrazione più decentrata della giustizia matrimoniale (soprattutto episcopale) non impone scelte tassative. Anche in questo caso l'ambizione della riforma richiede dunque il confronto e l'adattamento con le peculiarità e difficoltà locali. Non sembra però che l'esaurimento della procedura lontano dalla sede vescovile interessata possa soddisfare lo spirito e le esigenze del processo breviore ${ }^{101}$. Le affermazioni di principio e di valore vanno però adeguate alla conformazione tecnica del giudizio in questione. Un ulteriore problema è coniugare il richiamo papale con le risultanze del Tavolo di lavoro per l'Italia ${ }^{102}$. In questo contesto la designazione del Vicario giudiziale incaricato non sembra essere circoscritta al tribunale diocesano, si consente anche il ricorso all'Officiale interdiocesano, come di fatto praticato in diversi tribunali regionali o interdiocesani italiani ${ }^{103}$. L'autorevolezza pontificia si impone sulle soluzioni interpretative e applicative elaborate localmente ma non dovrebbe contrastare con l'indirizzo concordemente assunto dall'organismo specificamente costituito ${ }^{104}$. L'univocità del ministro nella materia matrimoniale può avere inoltre vantaggi pratici e funzionali ${ }^{105}$. $\mathrm{Al} \mathrm{di}$ là delle possibili discrasie (quantomeno congiunturali), il profilo organizza-

${ }^{100}$ N. 5 Discorso.

101 La figura del Vescovo è centrale nell'impostazione del giudizio. È vero che la decisione episcopale risulta essenzialmente come un giudizio sugli atti, ma non si vuole che l'ufficio capitale risulti o appaia come un mero firmatario o garante della sentenza, completamente avulso dall'introduzione della causa e dallo svolgimento dell'indagine.

102 «Per l'accesso al processo brevior, il Vicario giudiziale, al quale il Vescovo diocesano affidi l'esame del libello, valuta lo stesso in conformità ai criteri del Vescovo, al quale è ultimamente affidata la decisione» (Tavolo di lavoro CEI, n. 5).

103 Circa l'attuazione del Motu Proprio in alcuni tribunali italiani, cfr. La riforma del processo matrimoniale ad un anno dal Motu Proprio Mitis Iudex Dominus Iesus, 195-233. Per un inquadramento più generale della problematica: M. GANARIN, I tribunali interdiocesani secondo il m.p. Mitis Iudex Dominus Iesus. Riflessioni circa la "sorte" del m.p. Qua cura di Papa Pio XI, Stato, Chiese e pluralismo confessionale. Rivista telematica (www.statoechiese.it) n. 11 (2016) 1-24.

104 Il Tavolo di lavoro, coordinato dalla Segreteria della CEI, ha riunito il Prefetto del Supremo Tribunale della Segnatura Apostolica, il Presidente del Pontificio Consiglio per i Testi Legislativi e il Decano del Tribunale Apostolico della Rota Romana, appare quindi come un organo estremamente qualificato e autorevole, espressione di una proficua collaborazione istituzionale. La valenza delle conclusioni esplicitate dal Comunicato della CEI appare più generale e suscettibile di estensione ad altre realtà, per quanto richieda almeno un avallo o passaggio formale.

${ }^{105}$ Cfr. supra rif. nt. 97. Il deferimento dell'accesso al processo al Vicario interdiocesano, specie ove siano previste sezioni istruttorie locali o siano designati istruttori della diocesi di provenienza della causa, non appare contra voluntatem Legislatoris. 
torio richiede un'attuazione cauta e ponderata, evitando scelte precipitose e sbrigative ${ }^{106}$.

\section{LE ASPIRAZIONI E I RICHIAMI DI PRINCIPIO}

Vale la pena sottolineare infine che il Discorso del 25 novembre 2017 ha una portata direttiva più generale. Anche in linea con il desiderio di sensibilizzare e motivare il corpo episcopale, il Papa traccia una sorta di assiologia o quadro di riferimento valoriale del processus brevior. I tratti salienti della nuova figura processuale erano già stati enucleati nella formazione degli operatori e nella dottrina ${ }^{107}$, in questo contesto viene presentata una puntualizzazione o enfatizzazione sintetica e autorevole. Ci siamo già soffermati sulle coordinate della riforma segnalate (spirito sinodale, consolazione pastorale, centralità episcopale, supra $\$ 2$ ), in maniera più puntuale e mirata in vista dell'ermeneutica del processus brevior il Pontefice afferma: «La prossimità e la gratuità, come ho più volte ribadito, sono le due perle di cui hanno bisogno i poveri, che la Chiesa deve amare sopra ogni cosa». Emerge subito la coscienza della conferma o continuità dei valori indicati. Alla prossimità e gratuità, riteniamo si possa tranquillamente aggiungere la sollecitudine che caratterizza la forma processuale e viene ripetutamente ripresa. Conviene esaminare brevemente il significato e il contenuto di questi tre profili. Trattandosi di una sorta di riepilogo di idee già precedentemente accennate o esposte evitiamo eccessive ripetizioni o sovrapposizioni.

La sollecitudine indica non solo la rapidità e celerità che connotano il rito abbreviato ma la preoccupazione e cura che ispirano la figura del Vescovo-giudice. L'insistenza sulla prontezza della risposta di giustizia emerge in almeno tre passaggi. Anzitutto il Papa ha rammentato il criterio della concentrazione e

${ }^{106}$ Ad evidenziare la laboriosità dell'esecuzione della riforma già rilevavamo: «La "temporaneità" della concorrenza deriva dall'aspirazione (in tempi ragionevolmente piuttosto lunghi) a superare forme diverse dall'amministrazione diocesana della giustizia matrimoniale. La radicazione, diffusione e funzionalità dei tribunali interdiocesani non lascia presagire uno sviluppo rapido del sistema» (M. DEL Pozzo, I titoli di competenza e la "concorrenza materiale"..., cit., 474). Bisogna aver sempre presente che il processo ordinario continua ad essere il prototipo e la via principale di accertamento della nullità matrimoniale.

Il Sussidio applicativo ha fornito avvertenze e indicazioni esplicative del disposto. I Capisaldi della riforma individuano quattro punti: 1 . La centralità del Vescovo nel servizio della giustizia; 2. La sinodalità nel servizio pastorale della giustizia; 3 . Procedure più semplici e agili; 4 . La gratuità delle procedure (Sussidio applicativo MIDI, 9-12). La dottrina per lo più si è soffermata sulla semplicità, celerità, vicinanza ed economicità della nuova procedura. 
velocità istruttoria. È utile rilevare che la durata contenuta dell'istruttoria (che è l'anima del processo) è riconducibile alla prontezza e semplicità nell'acquisizione delle prove e non viceversa alla sommarietà e superficialità della ricerca. L'unicità dell'udienza non è comunque assoluta e dipende dalla laboriosità previa e contestuale alla fase probatoria ${ }^{108}$. Il desiderio di velocità connota peraltro l'attuazione stessa della forma processuale (quam primum). Lentezze o ritardi ingiustificati nell'accessibilità dello strumento, come accennato, manifestano, oltre che una scarsa obbedienza e docilità, anche un serio difetto di attenzione e disponibilità verso la pastorale familiare e i fedeli smarriti o sofferenti. La garanzia della sollecitudine è infine un'esigenza intrinseca alla giustizia matrimoniale. L'eventuale temporaneo rinvio generalizzato al processo ordinario non può eludere ma anzi spinge a rispettare comunque la brevità del giudizio ${ }^{109}$.

Lultimo rilievo evidenzia che la domanda di giustizia, nelle aspirazioni della riforma, deve poter trovare risposta ovunque in tempi rapidi. La prossimità si attua allora nelle materialità delle strutture, nella formazione del personale e, in maniera non meno importante, nell'atteggiamento degli operatori. La consolazione pastorale implica la considerazione premurosa della sofferenza, solitudine, ferita e povertà (morale o materiale) ${ }^{110}$ delle persone e, soprattutto, della pace e salvezza delle anime che sono al centro dell'accertamento giudiziario. La pregnanza del ruolo del Vescovo e la naturalità della sua funzione giudicante trovano quindi un riscontro nella responsabilità diretta $\mathrm{e}$ personale per l'amministrazione della giustizia nella propria Chiesa particolare. Il processus brevior esalta la presentazione della figura del Vescovo quale «padre, capo e giudice dei suoi fedeli». La vicinanza esprime allora la effettiva reperibilità e accessibilità del Pastore ${ }^{111}$. Il ricorso suppletorio e sussidiario alle strutture di un Vescovo viciniore ha perciò un carattere di straordinarietà e provvisorietà. Il dato che meglio evidenzia l'orientamento della riforma, soprattutto nel processus brevior, è la previsione di un autonomo tribunale e servizio giudiziario. La contiguità e prossimità dell'ufficio capitale rispetto agli istanti non deve essere solo formale e virtuale ma reale e sostanziale.

\footnotetext{
108 Il principio costante è il quam primum, salva iustitia (cfr. art. 72 DC).

109 Cfr. supra nt. 75.

110 Tali tratti sono espressamente menzionati nel Discorso.

111 Come abbiamo già indicato, la effettiva disponibilità sta nell'avveduta praticabilità e assicurazione del mezzo e non certo nella strumentale o disinvolta applicazione del processo abbreviato.
} 
La gratuità non riceve una specificazione attuativa o esemplificativa nel contesto del Discorso, è comunque una costante degli interventi pontifici ${ }^{112}$. L'espresso richiamo di principio in questa sede indica che il processus brevior dovrebbe distinguersi anche per l'economicità complessiva del giudizio. A prescindere dagli oneri monetari, il principale risparmio richiesto è quello strumentale e temporale. La gratuità non implica l'esenzione totale dalle spese, la povertà e l'indigenza ad ogni modo non possono essere mai una remora all'inoltro della domanda, meno che mai coram Episcopo. L'accessibilità può consistere dimostrativamente anche nella facilità o agevolazione dell'inoltro ${ }^{113}$.

\section{UN ULTERIORE APPORTO NEL CAMMINO DELLA RIFORMA}

Sarebbe lungo e probabilmente superfluo riepilogare i passaggi e le vicende della riforma in atto $^{114}$, il Discorso del 25 novembre 2017 si aggiunge a un insieme già abbastanza tormentato e complesso di documenti (per la difficoltà e spinosità della materia e il susseguirsi degli interventi), risolve e chiarisce alcuni punti, ingenera qualche ulteriore difficoltà o dubbio interpretativo $^{115}$, apre nuovi fronti alla discussione scientifica e all'approfondimento critico. Il contesto giudiziario ecclesiale (non troppo compatto e sedimentato) fa supporre che non sarà probabilmente l'ultima parola in merito allo stesso processo breviore né tantomeno alla riforma, resterà ad ogni modo un punto di riferimento obbligato e inderogabile. $\grave{E}$ sicuramente apprezzabile lo sforzo in prima persona del Pontefice per stimolare l'applicazione del processus brevior e garantire i diritti dei fedeli. Il tono e il contenuto dell'allocuzione manifestano soprattutto l'interesse e la determinazione per la corretta ricezione e attuazione della riforma processuale ${ }^{116}$. Il testo comunque non ci pare abbia un

112 Cfr. VI Criterio fondamentale, Proemio MIDI; FranCEsco, Rescritto, 7 dicembre 2015, II.6; IDEM, Rescritto, 22 gennaio 2016; IDEM, Discorso alla Rota Romana, 23 gennaio 2015.

113 Occorre sempre aver presente che la professionalità e la preparazione costano, ogni eventuale esonero o risparmio significa trovare fonti alternative di finanziamento. La responsabilizzazione dei fedeli è la prima e inesorabile via di soluzione (supra, Rescritto, I.6).

114 Cfr. l'ampia e analitica trattazione di G. BONI, La riforma del processo canonico di nullità matrimoniale... I-II-III; La recente riforma del processo di nullità matrimoniale... I-II-III.

115 I principali dubbi sono connessi alla modalità dell'istruttoria e alla menzione della Segnatura Apostolica.

${ }^{116}$ La corretta ricezione è uno dei passaggi più importanti del processo legislativo, cfr. anche J. OTADUY, Discernir la recepción. Las acepciones del concepto y su relieve en el derecho canónico, Fidelium Iura 7 (1997) 179-243. 
intento propriamente normativo e prescrittivo ma essenzialmente dichiarativo e interpretativo. Il Papa ha voluto ribadire la mens che ha guidato l'introduzione dell'istituto del processus brevior, sciogliere incertezze e contrasti e cercare di superare ostacoli e resistenze (concettuali e organizzative) all'applicazione. Il Discorso perciò, senza sminuirne la portata ermeneutica e prescrittiva, assume prevalentemente un valore d'indirizzo e di governo.

Fermo restando la nettezza e categoricità della formulazione delle singole affermazioni, ci sembra utile evidenziare che le parole del Pontefice devono essere accolte e comprese secondo il senso e lo scopo che le ha mosse e ispirate, evitando di attribuire un'influenza aggiuntiva o ultronea all'intervento papale. Le accezioni o qualificazioni usate sono sicuramente significative e indicative della voluntas Legislatoris, ma, salvo esplicita dichiarazione, non denotano un cambiamento o un'alterazione del testo legale. Il tono e lo stile, come evidenziato (supra $\$ 2$ ), possono magari confondere o sviare l'interprete. L'univoco testo di riferimento, stando alla stessa impostazione assunta, resta il dettato codiciale novellato. Non ci sembra corretto e rispondente attribuire un valore creativo o dirimente a talune espressioni adoperate nell'esposizione. A proposito delle condizioni di accesso al rito più breve il Papa precisa: «[...] richiedendosi come condizione imprescindibile l'assoluta evidenza dei fatti comprovanti la presunta nullità del coniugio, oltre al consenso dei due sposi». Il dato incontrovertibile è la concorrente e rigorosa verifica delle due condizioni ${ }^{117}$. La menzione della "assolutezza" dell'evidenza non riteniamo introduca una nuova e più restrittiva categoria, ma la limitazione tassativa dell'ipotesi alla manifesta nullità (che è già sufficientemente circoscritta ${ }^{118}$ ). La richiesta del consenso degli sposi sembra inclinare per una valutazione sostanziale e non solo processuale della posizione delle parti ma non comporta una automatica destituzione della tesi prospettata da alcuni autori ${ }^{119}$. Un dis-

117 La dottrina ha univocamente riconosciuto la necessaria concorrenza delle due condizioni.

118 Abbiamo già esposto come diversi autori ritengano la forma abbreviata straordinaria o abbastanza eccezionale, supra nt. 76.

119 Cfr. ad es. G. P. MONTINI, L'accordo dei coniugi quale presupposto del "processus matrimonialis brevior" (can. 1683, $1^{\circ}$ MI), Periodica 105 (2016) 395-415; L. SABBARESE, Il processo più breve: condizioni per la sua introduzione, procedura, decisione, in E. B. O. ОKОNKWO - A. RECCHIA (eds.), Tra rinnovamento e continuità. Le riforme introdotte dal motu proprio Mitis Iudex Dominus Iesus, Urbaniana University Press, Città del Vaticano 2016, 46-49. Riteniamo peraltro che tale tesi sia contraria allo spirito e all'impostazione della legge. La formulazione del Discorso avvalora l'interpretazione maggioritaria e diretta ma non si propone di risolvere esplicitamente detta questione (probabilmente ritenuta già chiara e scontata). 
corso simile vale anche per la potestas decidendi del Metropolita, del Suffraganeo più anziano o del Decano in merito all'appello. Il riferimento al rigetto o all'ammissione (previsti peraltro dallo stesso testo legale) suffraga un'operazione diversa dalla convalida ma non precisa il contenuto della verifica ${ }^{120}$. Le esemplificazioni si potrebbero anche ampliare. Un eccessivo e troppo spinto "letteralismo ermeneutico" risulta improprio, strumentale e contrario alla razionalità e organicità del sistema.

Le considerazioni proposte sono evidentemente riflessioni provvisorie e in itinere, soprattutto per quanto riguarda gli aspetti incerti o ancora aperti all'integrazione attuativa (supra $\$ 5$ ). L'intervento, come abbiamo cercato di evidenziare, ha una prospettiva generale e un carattere interpretativo abbastanza differenziato. L'attenzione prevalente sulle puntualizzazioni analitiche relative al processus brevior non deve far perdere di vista l'orizzonte di senso e di valore complessivo delineato, nonché lo spirito direttivo e la modalità chiarificatrice dell'apporto. Il processo breviore si inserisce nel contesto della riforma e ne rappresenta solo un aspetto (non si allontana probabilmente dalla classica "punta dell'iceberg"), la fedeltà alle prescrizioni dei Motu Proprio è però rappresentativa della effettiva disponibilità e coesione del corpo episcopale. L'obiettivo finale (di medio-lunga portata) resta sempre l'assicurazione "ordinaria" e generalizzata di una giustizia matrimoniale solerte ed efficiente che supera le possibilità del solo Vescovo diocesano giudice ${ }^{121}$.

I destinatari più diretti dell'allocuzione risultano invero i Vescovi diocesani. Il fattivo concorso del corpo episcopale all'attuazione della riforma è la dimostrazione dello spirito sinodale e della conversione istituzionale e personale sollecitata in materia più che dal Papa dal popolo cristiano ${ }^{122}$. La flessibilità e duttilità della forma abbreviata non si concreta però in una disponibilità e

120 Cfr. supra nt. 69.

121 Il fattore temporale e l' "investimento" (economico e materiale) nella formazione è una componente necessaria della preparazione degli operatori (Vescovo compreso). Cfr. anche M. DEL Pozzo, Il profilo del giudice ecclesiastico (commento al Discorso alla Rota Romana del 2014), Ius Ecclesiae 26 (2014) 473-484.

122 È indicativa della consapevolezza e responsabilità dell'episcopato in merito alla riforma del processo matrimoniale la proposizione n. 82 della Relazione finale (24 ottobre 2015) della XVI AsSEMBLEA GENERALE ORDINARIA DEL SINODO DEI VESCOVI: «L'attuazione di questi documenti costituisce dunque una grande responsabilità per gli Ordinari diocesani, chiamati a giudicare loro stessi alcune cause e, in ogni modo, ad assicurare un accesso più facile dei fedeli alla giustizia. Ciò implica la preparazione di un personale sufficiente, composto di chierici e laici, che si consacri in modo prioritario a questo servizio ecclesiale» («L'Osservatore Romano», 26-27 ottobre 2015). 
indeterminatezza dello strumento processuale ${ }^{123}$. I chiarimenti, pur lasciando una certa ampiezza (soprattutto riguardo alla gestione della fase istruttoria) e rimettendosi alla valutazione e preparazione dei singoli Pastori, si muovono nella linea di definire univocamente criteri e condizioni della procedura. La responsabilità e il coinvolgimento del Vescovo richiesti non riguardano soltanto l'impegno personale ma la preoccupazione e la qualità del servizio giudiziario predisposto. Ribadiamo ancora una volta che la sfida del nuovo processo (compreso il processus brevior) concerne soprattutto l'incremento e la formazione dei ministri, dei patroni e dei consulenti familiari ${ }^{124}$. L'inconsueta e singolare modalità della "interpretazione autentica" resa denota la necessità di ovviare ad una certa sofferenza e difficoltà nell'esecuzione del disposto. Non può che auspicarsi pertanto un maggior coordinamento e dialogo istituzionale e un recupero dei ruoli e delle competenze che conduca al sereno assestamento della situazione ${ }^{125}$.

${ }^{123}$ La formula «in conformità ai criteri del Vescovo diocesano» (Sussidio applicativo MIDI, 24 e 25), ripresa anche dal Tavolo di lavoro CEI (n. 5) può dar luogo a equivoci o fraintendimenti.

${ }^{124}$ Cfr. M. DEL Pozzo, L'organizzazione giudiziaria ecclesiastica alla luce del m. p. "Mitis iudex", Stato, Chiese e pluralismo confessionale, Rivista telematica (www.statoechiese.it) n. 36 (2015) 2933; IDEM, L'impatto della riforma..., cit., 79-80.

125 Il ruolo della Segnatura Apostolica e del Pontificio Consiglio per i Testi Legislativi appare abbastanza dimesso e defilato in questa fase così cruciale e delicata per l'implementazione della riforma processuale. 


\section{Bibliografía}

Arroba Conde, M. J. - IzZI, C., Pastorale giudiziaria e prassi processuale nelle cause di nullità del matrimonio. Dopo la riforma operata con il motu proprio Mitis Iudex Dominus Iesus, Edizioni San Paolo, Cinisello Balsamo 2017.

Badisseri, L. (ed.), A cinquant'anni dall'Apostolica Sollicitudo. Il Sinodo dei Vescovi al servizio di una Chiesa sinodale. Atti del Seminario di studio organizzato dalla Segreteria generale del Sinodo dei Vescovi (Città del Vaticano, 6-9 febbraio 2016), LEV, Città del Vaticano 2016.

BAURA, E., Il valore normativo dell'Istruzione "Dignitas connubii", in P. A. BONNET - C. Gullo (eds.), Il giudizio di nullità matrimoniale dopo l'istruzione «Dignitas connubii». Parte Prima: i principi, LEV, Città del Vaticano 2007, 185-211.

BELFIORE, G., I processi di nullità matrimoniale nella riforma di papa Francesco, Edizioni Grafiser-Troina, Catania 2017.

BenedetTo XVI, cost. ap. Anglicanorum coetibus, 4-XI-2009.

BIANCHI, P., Il processus brevior coram Episcopo. Le prime esperienze di un tribunale locale, in AA. Vv., Studi in onore di Carlo Gullo, III, LEV, Città del Vaticano 2017, 799-826.

—, Lo svolgimento del processo breve: la fase istruttoria e di discussione della causa, in REDAZIONE DI QUADERNI DI DIRITTO ECCLESIALE (ed.), La riforma dei processi matrimoniali di papa Francesco. Una guida per tutti, Àncora, Milano 2016, 67-90.

BONI, G., La riforma del processo canonico di nullità matrimoniale: il complicarsi progressivo del quadro delle fonti normative (parte prima, seconda e terza), Stato, Chiese e pluralismo confessionale. Rivista telematica (www.statoechiese.it) nn. 4-6 (2018), 1-78, 1-103, 1-29.

—, La recente riforma del processo di nullità matrimoniale. Problemi, criticità, dubbi (parte prima, seconda e terza), Stato, Chiese e pluralismo confessionale. Rivista telematica (www.statoechiese.it) nn. 11, 4-5 (2016-2017) 1-112, 169, 1-82.

Bunge, A. W., Presentación del nuevo proceso matrimonial, Anuario Argentino de Derecho Canónico 21 (2015) 73-75.

Conferenza Episcopale Italiana, Testo del tavolo di lavoro, 20-VII-2016.

Daniel, W. L., The Abbreviated Matrimonial Process before the Bishop in Cases of "Manifest Nullity" of Marriage, The Jurist 75 (2015) 539-591.

Del Pozzo, M., La centralità della coscienza nella verità del matrimonio, Ius Ecclesiae 30 (2018) 367-380. 
—, L'appello nel 'processus matrimonialis brevior', Archivio Giuridico 237/3-4 (2017) 485-535.

—, I titoli di competenza e la "concorrenza materiale" alla luce del m. p. Mitis iudex Dominus Iesus, Ius Ecclesiae 28 (2016) 457-478.

—, Il processo matrimoniale più breve davanti al Vescovo, EDUSC, Roma 2016.

-, Considerazioni sui ricorsi della fase introduttiva del giudizio matrimoniale nell'impianto del m. p. "Mitis iudex", Stato, Chiese e pluralismo confessionale, Rivista telematica (www.statoechiese.it) n. 34 (2016) 1-24.

—, L'organizzazione giudiziaria ecclesiastica alla luce del m. p. "Mitis iudex", Stato, Chiese e pluralismo confessionale, Rivista telematica (www.statoechiese.it) n. 36 (2015) 1-33.

- Il profilo del giudice ecclesiastico (commento al Discorso alla Rota Romana del 2014), Ius Ecclesiae 26 (2014) 473-484.

FranCESCO, Discorso in occasione dell'inaugurazione dell'anno giudiziario del Tribunale della Rota Romana, 29-I-2018.

—, Discorso ai partecipanti al corso promosso dal Tribunale della Rota Romana, 25XI-2017.

—, Discorso ai partecipanti al corso promosso dal Tribunale della Rota Romana, 12III-2016, Quaderni dello Studio rotale 9 (2016) 49-52.

Ganarin, M., I tribunali interdiocesani secondo il m.p. Mitis Iudex Dominus Iesus. Riflessioni circa la "sorte" del m.p. Qua cura di Papa Pio XI, Stato, Chiese e pluralismo confessionale. Rivista telematica (www.statoechiese.it) n. 11 (2016) 1-24.

—, Linterpretazione autentica delle leggi universali della Chiesa, Bononia University Press, Bologna 2016.

—, Il Pontificio Consiglio per i Testi Legislativi nell'assetto istituzionale della Curia Romana tra diritto vigente e prospettive di riforma, Stato, Chiese e pluralismo confessionale. Rivista telematica (www.statoechiese.it) n. 22 (2015) 1-76.

Ghirlanda, G., Il diritto nella Chiesa mistero di comunione. Compendio di diritto ecclesiale, Gregorian \& Biblical Press, Roma 2014.

Giovanni PaOlo II, cost. ap. Ecclesia in Urbe, 1-I-1998.

—, cost. ap. Spiritalium militum curae, 24-IV-1986.

Llobell, J., I processi matrimoniali nella Chiesa, EDUSC, Roma 2015.

-, Processi e procedure penali: sviluppi recenti, in H. FRANCESCHI - M. Á. ORTIZ (eds.), Ius et matrimonium. Temi di diritto matrimoniale e processuale canonico, EDUSC, Roma 2015, 75-108. 
—, La certezza morale nel processo canonico, Il Diritto Ecclesiastico 109/1 (1998) 758-802.

-, La natura giuridica e la recezione ecclesiale dell'istr. «Dignitas connubii», Ius Ecclesiae 18 (2006) 344-354.

-, Sulla valenza giuridica dei discorsi del Romano Pontefice al Tribunale Apostolico della Rota Romana, L'Osservatore Romano 6-XI-2005, 7-8.

MontinI, G. P., Gli elementi pregiudiziali del processus brevior: consenso delle parti e chiara evidenza di nullità, in AA. Vv., Prassi e sfide dopo l'entrata in vigore del m.p. Mitis Iudex Dominus Iesus e del Rescriptum ex audientia del 7 dicembre 2015, LEV, Città del Vaticano 2017, 47-64.

—, «Si appellatio mere dilatoria evidenter appareat» (cann. 1680 \$ 2 e 1687 S 4 MIDI): alcune considerazioni, Periodica 105 (2016) 663-699.

—, L'accordo dei coniugi quale presupposto del "processus matrimonialis brevior" (can. 1683, $1^{\circ}$ MI), Periodica 105 (2016) 395-415.

Morán Bustos, C. M., El proceso "brevior" ante el Obispo diocesano, in M. E. Olmos Ortega (ed.), Procesos de nulidad matrimonial tras la reforma del Papa Francisco, Dykinson, Madrid 2016, 125-175.

Musso, L. - Fusco, C. (eds.), La riforma del processo matrimoniale ad un anno dal Motu Proprio Mitis Iudex Dominus Iesus, LEV, Città del Vaticano 2017.

OTADUY, J., Discernir la recepción. Las acepciones del concepto y su relieve en el derecho canónico, Fidelium Iura 7 (1997) 179-243.

PAOlO VI, m. p. Catholica Ecclesia, 23-X-1976, n. 4, AAS 68 (1976) 696.

PEÑa GaRCía, C., La reforma de los procesos canónicos de nulidad matrimonial: el motu proprio «Mitis Iudex Dominus Iesus», Estudios Eclesiásticos 90 (2015) 621-682.

PINTO, P. V., Matrimonio y familia en el camino sinodal del Papa Francisco, Arquidiócesis de Bogotá 2017, pro manuscripto, (Processus brevior del Papa Francisco Obispo de Roma, 13-VII-2017), 278-283.

RaBINO, G., Riflessioni sull'origine sinodale del processus brevior coram Episcopo, Stato, Chiese e pluralismo confessionale. Rivista telematica (www.statoechiese.it) n. 33 (2017) 1-24.

Romana, coram Augustino Card. Vallini (Vicario di Sua Santità Papa Francesco per la Diocesi di Roma), sentenza, 9-XII-2016, Prot. 17.830, in AA. Vv., Prassi e sfide dopo l'entrata in vigore del m.p. Mitis Iudex Dominus Iesus e del Rescriptum ex audientia del 7 dicembre 2015, LEV, Città del Vaticano 2017, 151-157.

Romana coram Francisco P.P., sentenza, 6-V-2017, Prot. 18.067, in AA. Vv., Prassi e sfide dopo l'entrata in vigore del m.p. Mitis Iudex Dominus Iesus e del 
Rescriptum ex audientia del 7 dicembre 2015, LEV, Città del Vaticano 2017, 141-149.

SABBARESE, L., Il processo più breve: condizioni per la sua introduzione, procedura, decisione, in E. B. O. OKONKWO - A. RECCHIA (eds.), Tra rinnovamento e continuità. Le riforme introdotte dal motu proprio Mitis Iudex Dominus Iesus, Urbaniana University Press, Città del Vaticano 2016, 39-58.

STANKIEWICZ, A., Alcune considerazioni intorno all'esercizio personale e vicario della ptestà giudiziale con riferimento al processo matrimoniale breviore davanti al Vescovo, in AA. Vv., Studi in onore di Carlo Gullo, III, LEV, Città del Vaticano 2017, 761-775.

-, La certezza morale e la motivazione della sentenza, in H. FRANCESCHI - J. LLOBELL - M. Á. ORTIZ (eds.), La nullità del matrimonio: temi processuali e sostantivi in occasione della «Dignitas Connubii», EDUSC, Roma 2005, 231-245.

Supremo Tribunale della Segnatura Apostolica, Lettera circolare "Inter munera" sullo stato e l'attività dei Tribunali, 30-VII-2016, Prot. n. 51712/16 VT.

Tribunale Apostolico della Rota Romana, Sussidio applicativo del Motu pr. Mitis Iudex Dominus Iesus, Città del Vaticano, gennaio 2016.

VIANA, A., Sinodalidad y derecho canónico. Un seminario organizado por la Secretaría general del Sínodo de los Obispos, Estudios eclesiásticos 92 (2017) 683-701.

—, «Ordinariato militar», in J. OTAduY - A. Viana - J. Sedano (eds.), Diccionario General de Derecho Canónico, V, Thomson Reuters Aranzadi, Cizur Menor (Navarra) 2012, 810.

Yunga Ndosimau, J., Les actes judiciaires dans la structure de la certitude morale à la lumiere de l'art. 247 de la Dignitas connubii, Médiaspaul, Kinshasa 2011. 
